\title{
Fejér-Pachpatte-Mercer-Type Inequalities for Harmonically Convex Functions Involving Exponential Function in Kernel
}

\author{
Saad Ihsan Butt $\mathbb{D}^{1},{ }^{1}$ Saba Yousaf $\mathbb{D}^{1},{ }^{1}$ Khuram Ali Khan $\mathbb{D}^{2},{ }^{2}$ Rostin Matendo Mabela $\mathbb{D},{ }^{3}$ \\ and Abdullah M. Alsharif $\mathbb{i D}^{4}$ \\ ${ }^{1}$ Department of Mathematics, COMSATS University Islamabad, Islamabad, Lahore Campus, Pakistan \\ ${ }^{2}$ Department of Mathematics, University of Sargodha, Sargodha 40100, Pakistan \\ ${ }^{3}$ Department of Maths and Computer Science, University of Kinshasa, Democratic Republic of the Congo \\ ${ }^{4}$ Department of Mathematics and Statistics, College of Science, Taif University, P. O. Box 11099, Taif 21944, Saudi Arabia
}

Correspondence should be addressed to Rostin Matendo Mabela; rostin.mabela@unikin.ac.cd

Received 17 October 2021; Accepted 14 December 2021; Published 2 March 2022

Academic Editor: Muhammad Shoaib Anwar

Copyright (c) 2022 Saad Ihsan Butt et al. This is an open access article distributed under the Creative Commons Attribution License, which permits unrestricted use, distribution, and reproduction in any medium, provided the original work is properly cited.

\begin{abstract}
In the present study, fractional variants of Hermite-Hadamard, Hermite-Hadamard-Fejér, and Pachpatte inequalities are studied by employing Mercer concept. Firstly, new Hermite-Hadamard-Mercer-type inequalities are presented for harmonically convex functions involving fractional integral operators with exponential kernel. Then, weighted Hadamard-Fejér-Mercer-type inequalities involving exponential function as kernel are proved. Finally, Pachpatte-Mercer-type inequalities for products of harmonically convex functions via fractional integral operators with exponential kernel are constructed.
\end{abstract}

\section{Introduction}

Integral inequalities have been widely used in various sciences, including mathematical sciences, applied sciences, differential equations, and functional analysis. In the last two decades, these inequalities have gained attention from researchers. In most mathematical analysis areas, many types of integral inequalities are used. They are very important in approximation theory and numerical analysis, which estimate the error's approximation. Integral inequalities are useful tools in the study of different classes of differential equations and integral equations. They are today employed not only in mathematics but also in physics, computer science, and biology.

Convexity has several uses in business, medicine, industry, and art that have a significant impact on our daily lives. One of the most important applications of the convex function is the formulation of inequalities. Many equalities and inequalities have been defined for convex functions, but Jensen's inequality and the Hermite-Hadamard integral inequality are the most notable results [1-3]. The following notion of convex function plays a significant role in optimization theory and in other fields of sciences.

Definition 1. A function $\Omega: I \subset \mathbb{R} \longrightarrow \mathbb{R}$ is called a convex function on $I$, if

$$
\Omega\left(\eta \mathfrak{a}_{1}+(1-\eta) \mathfrak{a}_{2}\right) \leq \eta \Omega\left(\mathfrak{a}_{1}\right)+(1-\eta) \Omega\left(\mathfrak{a}_{2}\right),
$$

for all $\mathfrak{a}_{1}, \mathfrak{a}_{2} \in I$ and $\eta \in[0,1]$, holds.

The following classical Jensen's inequality is defined as generalization of convex functions.

Theorem 1 (see [1]). Suppose that $\Omega$ is a convex function on $\left[\mathfrak{a}_{1}, \mathfrak{a}_{2}\right]$; then,

$$
\Omega\left(\sum_{\ell=1}^{n} \eta_{\ell} \varkappa_{\ell}\right) \leq \sum_{\ell=1}^{n} \eta_{\ell} \Omega\left(\varkappa_{\ell}\right),
$$

for all $\varkappa_{\ell} \in\left[\mathfrak{a}_{1}, \mathfrak{a}_{2}\right]$ and $\eta_{\ell} \in[0,1]$, where $\ell=1,2, \ldots, n$ with $\sum_{\ell=1}^{n} \eta_{\ell}=1$. 
Inequality (2) is a key to extract applications in information theory. It is very useful in computing optimal bounds for joint and conditional entropies and mutual information (for instance, see [4-6] and the references therein).

Hermite-Hadamard inequalities may be considered as a refinement of the concept of convexity, and it is simply inferred from Jensen's inequality as follows.

Theorem 2 (see [2]). If $\Omega$ is a convex function on the interval $\left[\mathfrak{a}_{1}, \mathfrak{a}_{2}\right]$ with $\mathfrak{a}_{1}<\mathfrak{a}_{2}$, then

$$
\Omega\left(\frac{\mathfrak{a}_{1}+\mathfrak{a}_{2}}{2}\right) \leq \frac{1}{\mathfrak{a}_{2}-\mathfrak{a}_{1}} \int_{\mathfrak{a}_{1}}^{\mathfrak{a}_{2}} \Omega(\varkappa) \mathrm{d} \varkappa \leq \frac{\Omega\left(\mathfrak{a}_{1}\right)+\Omega\left(\mathfrak{a}_{2}\right)}{2} .
$$

It has been applied in several branches such as finance, engineering, and science (see [2]). In recent years, Hermite-Hadamard inequalities for convex functions have gotten a lot of attention, and as a result, there have been a lot of refinements and generalizations.

In 2003, Mercer presented a variant of Jensen's inequality as follows.

Theorem 3 (see [7]). Suppose that $\Omega$ is a convex function on the interval $\left[\theta_{1}, \theta_{2}\right]$; then,

$\Omega\left(\theta_{1}+\theta_{2}-\sum_{\ell=1}^{n} \eta_{\ell} \varkappa_{\ell}\right) \leq \Omega\left(\theta_{1}\right)+\Omega\left(\theta_{2}\right)-\sum_{\ell=1}^{n} \eta_{\ell} \Omega\left(\varkappa_{\ell}\right)$,

for all $\varkappa_{\ell} \in\left[\theta_{1}, \theta_{2}\right]$ and $\eta_{\ell} \in[0,1]$, where $\ell=1,2, \ldots, n$ with $\sum_{\ell=1}^{n} \eta_{\ell}=1$.

Over the years, the Jensen-Mercer inequality became a topic of foremost interest for many scholars as they have investigated and studied in various ways including bringing it to a higher dimension and acquiring it for convex operators along with its several refinements, operator variants for superquadratic functions, improvements, and many generalizations with applications in information theory (see [8-10]).

In [11], Íşcan gave the definition of harmonic convexity as follows.

Definition 2. A function $\Omega: I \subset \mathbb{R} \backslash\{0\} \longrightarrow \mathbb{R}$ is said to be harmonically convex on $I$, if

$$
\Omega\left(\frac{\mathfrak{a}_{1} \mathfrak{a}_{2}}{\eta \mathfrak{a}_{1}+(1-\eta) \mathfrak{a}_{2}}\right) \leq \eta \Omega\left(\mathfrak{a}_{2}\right)+(1-\eta) \Omega\left(\mathfrak{a}_{1}\right),
$$

holds for all $\mathfrak{a}_{1}, \mathfrak{a}_{2} \in I$ and $\eta \in[0,1]$.

In [11], Íşcan for the first time introduced the Hermite-Hadamard inequality for harmonically convex function along with the following identity.

Theorem 4 (see $[11]$ ). Suppose that $\Omega: I \subseteq(0, \infty) \longrightarrow \mathbb{R}$ is a harmonically convex function and $\mathfrak{a}_{1}, \mathfrak{a}_{2} \in I$ with $\mathfrak{a}_{1}<\mathfrak{a}_{2}$. If $\Omega \in L\left[\mathfrak{a}_{1}, \mathfrak{a}_{2}\right]$, then

$$
\Omega\left(\frac{2 \mathfrak{a}_{1} \mathfrak{a}_{2}}{\mathfrak{a}_{1}+\mathfrak{a}_{2}}\right) \leq \frac{\mathfrak{a}_{1} \mathfrak{a}_{2}}{\mathfrak{a}_{2}-\mathfrak{a}_{1}} \int_{\mathfrak{a}_{1}}^{\mathfrak{a}_{2}} \frac{\Omega(\varkappa)}{\varkappa^{2}} \mathrm{~d} \varkappa \leq \frac{\Omega\left(\mathfrak{a}_{1}\right)+\Omega\left(\mathfrak{a}_{2}\right)}{2} .
$$

Lemma 1 (see [11]). If $\Omega: I \subseteq(0, \infty) \longrightarrow \mathbb{R}$ is a differentiable function on $I^{o}$ and $\mathfrak{a}_{1}, \mathfrak{a}_{2} \in I$ with $\mathfrak{a}_{1}<\mathfrak{a}_{2}$ and $\Omega^{\prime} \in L\left[\mathfrak{a}_{1}, \mathfrak{a}_{2}\right]$, then

$$
\begin{aligned}
& \frac{\Omega\left(\mathfrak{a}_{1}\right)+\Omega\left(\mathfrak{a}_{2}\right)}{2}-\frac{\mathfrak{a}_{1} \mathfrak{a}_{2}}{\mathfrak{a}_{2}-\mathfrak{a}_{1}} \int_{\mathfrak{a}_{1}}^{\mathfrak{a}_{2}} \frac{\Omega(\varkappa)}{\varkappa^{2}} \mathrm{~d} \varkappa \\
& =\frac{\mathfrak{a}_{1} \mathfrak{a}_{2}\left(\mathfrak{a}_{2}-\mathfrak{a}_{1}\right)}{2} \int_{0}^{1} \frac{1-2 \eta}{\left(\eta \mathfrak{a}_{2}+(1-\eta) \mathfrak{a}_{1}\right)^{2}} \Omega^{\prime} \\
& \cdot\left(\frac{\mathfrak{a}_{1} \mathfrak{a}_{2}}{\eta \mathfrak{a}_{2}+(1-\eta) \mathfrak{a}_{1}}\right) \mathrm{d} \eta .
\end{aligned}
$$

The most prominent inequalities connected to the integral mean of a harmonically convex function are the Hermite-Hadamard inequalities or their weighted versions which are called Hermite-Hadamard-Fejér inequalities for harmonically convex functions. In [12], Chen and $\mathrm{Wu}$ established the Hermite-Hadamard-Fejér inequality for harmonically convex functions.

Theorem 5 (see [12]). Let $\Omega: I \subseteq(0, \infty) \longrightarrow \mathbb{R}$ be a harmonically convex function and $\mathfrak{a}_{1}, \mathfrak{a}_{2} \in I$ with $\mathfrak{a}_{1}<\mathfrak{a}_{2}$. If $\Omega \in L\left[\mathfrak{a}_{1}, \mathfrak{a}_{2}\right]$ and $\omega:\left[\mathfrak{a}_{1}, \mathfrak{a}_{2}\right] \subseteq(0, \infty) \longrightarrow \mathbb{R}$ is non-negative, integrable, and harmonically symmetric with respect to $2 \mathfrak{a}_{1} \mathfrak{a}_{2} / \mathfrak{a}_{1}+\mathfrak{a}_{2}$, that is, $\omega(\varkappa)=\omega\left(1 /\left(1 / \mathfrak{a}_{1}\right)+\left(1 / \mathfrak{a}_{2}\right)-(1 / \varkappa)\right)$, then

$$
\begin{aligned}
\Omega\left(\frac{2 \mathfrak{a}_{1} \mathfrak{a}_{2}}{\mathfrak{a}_{1}+\mathfrak{a}_{2}}\right) \int_{\mathfrak{a}_{1}}^{\mathfrak{a}_{2}} \frac{\omega(\varkappa)}{\varkappa^{2}} \mathrm{~d} \varkappa & \leq \int_{\mathfrak{a}_{1}}^{\mathfrak{a}_{2}} \frac{\Omega(x) \omega(\varkappa)}{\varkappa^{2}} \mathrm{~d} \varkappa \\
& \leq \frac{\Omega\left(\mathfrak{a}_{1}\right)+\Omega\left(\mathfrak{a}_{2}\right)}{2} \int_{\mathfrak{a}_{1}}^{\mathfrak{a}_{2}} \frac{\omega(\varkappa)}{\varkappa^{2}} \mathrm{~d} \varkappa .
\end{aligned}
$$

In [13], Chen and $\mathrm{Wu}$ obtained two Hermite-Hadamard-type inequalities for products of harmonically convex functions as follows.

Theorem 6 (see [13]). Let $\Omega, \omega:\left[\mathfrak{a}_{1}, \mathfrak{a}_{2}\right] \subseteq(0, \infty) \longrightarrow$ $[0, \infty), \quad \mathfrak{a}_{1}, \mathfrak{a}_{2} \in(0, \infty), \quad$ be functions such that $\Omega, \omega, \Omega \omega \in L\left[\mathfrak{a}_{1}, \mathfrak{a}_{2}\right]$. If $\Omega$ and $\omega$ are harmonically convex on $\left[\mathfrak{a}_{1}, \mathfrak{a}_{2}\right]$, then

$$
\begin{aligned}
\frac{\mathfrak{a}_{1} \mathfrak{a}_{2}}{\mathfrak{a}_{2}-\mathfrak{a}_{1}} \int_{\mathfrak{a}_{1}}^{\mathfrak{a}_{2} \Omega(\varkappa) \omega(\varkappa)} \mathrm{d} \varkappa \leq & \frac{1}{3} M\left(\mathfrak{a}_{1}, \mathfrak{a}_{2}\right)+\frac{1}{6} N\left(\mathfrak{a}_{1}, \mathfrak{a}_{2}\right), \\
2 \Omega\left(\frac{2 \mathfrak{a}_{1} \mathfrak{a}_{2}}{\mathfrak{a}_{1}+\mathfrak{a}_{2}}\right) \omega\left(\frac{2 \mathfrak{a}_{1} \mathfrak{a}_{2}}{\mathfrak{a}_{1}+\mathfrak{a}_{2}}\right) \leq & \frac{\mathfrak{a}_{1} \mathfrak{a}_{2}}{\mathfrak{a}_{2}-\mathfrak{a}_{1}} \int_{\mathfrak{a}_{1}}^{\mathfrak{a}_{2}} \frac{\Omega(\varkappa) \omega(\varkappa)}{\varkappa^{2}} \mathrm{~d} \varkappa \\
& +\frac{1}{6} M\left(\mathfrak{a}_{1}, \mathfrak{a}_{2}\right)+\frac{1}{3} N\left(\mathfrak{a}_{1}, \mathfrak{a}_{2}\right),
\end{aligned}
$$

where $M\left(\mathfrak{a}_{1}, \mathfrak{a}_{2}\right)=\Omega\left(\mathfrak{a}_{1}\right) \omega\left(\mathfrak{a}_{1}\right)+\Omega\left(\mathfrak{a}_{2}\right) \omega\left(\mathfrak{a}_{2}\right)$ and $N\left(\mathfrak{a}_{1}\right.$, $\left.\mathfrak{a}_{2}\right)=\Omega\left(\mathfrak{a}_{1}\right) \omega\left(\mathfrak{a}_{2}\right)+\Omega\left(\mathfrak{a}_{2}\right) \omega\left(\mathfrak{a}_{1}\right)$. 
Fractional calculus is an extension of classical calculus. Fractional calculus is now a well-known technique in engineering science, with wide range of applications in material modeling. A growing number of degree considerations have recently been given to fractional calculus and its numerous applications. Fractional integral/derivative operators are extremely important in the development of fractional calculus. Fractional differential equations and dynamical frameworks were established a few decades ago as key tools for exhibiting a variety of phenomena in various branches of pure and applied sciences. Many physical problems can be modeled using fractional differential equations, including heat equations, wave equations, Poisson equations, and Laplace equations, biological populations, fluid mechanics, thermodynamics, viscoelasticity, vibration, advection-diffusion, groundwater flow with memory, and signal processing [14, 15]. Several studies have shown that fractional operators can accurately explain complex long memory and multiscale phenomena in materials that are difficult to capture using standard mathematical methods including classical differential calculus. The significance of fractional calculus can be more understandable, and several works involving fractional calculus have been done.
Several well-known inequalities and related results can be generalized and extended via fractional integral operators (see [16-19] and the references therein).

Definition 3 (see [15]). Let $\Omega \in L\left[\mathfrak{a}_{1}, \mathfrak{a}_{2}\right]$. The Riemann-Liouville fractional integrals $J_{\mathfrak{a}_{1}^{+}}^{\alpha} \Omega$ and $J_{\mathfrak{a}_{2}^{-}}^{\alpha} \Omega$ of order $\alpha>0$ with $\mathfrak{a}_{1} \geq 0$ are defined by

$$
\begin{aligned}
& J_{\mathfrak{a}_{1}^{+}}^{\alpha} \Omega(\varkappa)=\frac{1}{\Gamma(\alpha)} \int_{\mathfrak{a}_{1}}^{\varkappa}(\varkappa-u)^{\alpha-1} \Omega(u) \mathrm{d} u, \quad \varkappa>\mathfrak{a}_{1}, \\
& J_{\mathfrak{a}_{2}^{-}}^{\alpha} \Omega(\varkappa)=\frac{1}{\Gamma(\alpha)} \int_{\varkappa}^{\mathfrak{a}_{2}}(u-\varkappa)^{\alpha-1} \Omega(u) \mathrm{d} u, \quad \varkappa<\mathfrak{a}_{2},
\end{aligned}
$$

respectively. Here, $\Gamma(\alpha)$ is the gamma function defined by $\Gamma(\alpha)=\int_{0}^{\infty} e^{-u} u^{\alpha-1} \mathrm{~d} u$ and $J_{\mathfrak{a}_{1}^{+}}^{0} \Omega(\varkappa)=J_{\mathfrak{a}_{2}^{-}}^{0} \Omega(\varkappa)=\Omega(\varkappa)$.

In [20], Iscan and $\mathrm{Wu}$ for the first time introduced Hermite-Hadamard-type inequalities for harmonically convex functions for Riemann-Liouville fractional integral operators along with the following integral identity.

Theorem 7 (see [20]). Let $\Omega:\left[\mathfrak{a}_{1}, \mathfrak{a}_{2}\right] \subseteq(0, \infty) \longrightarrow \mathbb{R}$ be $a$ function with $\mathfrak{a}_{1}<\mathfrak{a}_{2}$ and $\Omega \in L\left[\mathfrak{a}_{1}, \mathfrak{a}_{2}\right]$. If $\Omega$ is a harmonically convex function, then

$$
\Omega\left(\frac{2 \mathfrak{a}_{1} \mathfrak{a}_{2}}{\mathfrak{a}_{1}+\mathfrak{a}_{2}}\right) \leq \frac{\Gamma(\alpha+1)}{2}\left(\frac{\mathfrak{a}_{1} \mathfrak{a}_{2}}{\mathfrak{a}_{2}-\mathfrak{a}_{1}}\right)^{\alpha}\left[J_{1 / \mathfrak{a}_{1}^{-}}^{\alpha}(\Omega \circ \mathfrak{g})\left(\frac{1}{\mathfrak{a}_{2}}\right)+I_{1 / \mathfrak{a}_{2}^{+}}^{\alpha}(\Omega \circ \mathfrak{g})\left(\frac{1}{\mathfrak{a}_{1}}\right)\right] \leq \frac{\Omega\left(\mathfrak{a}_{1}\right)+\Omega\left(\mathfrak{a}_{2}\right)}{2}
$$

where $\mathfrak{g}(\mathfrak{t})=1 / \mathfrak{t}$.

Lemma 2 (see [20]). Suppose that $\Omega:\left[\mathfrak{a}_{1}, \mathfrak{a}_{2}\right] \subseteq$ $(0, \infty) \longrightarrow \mathbb{R}$ is a differentiable function on $I^{o}$ and $\Omega^{\prime} \in L\left[\mathfrak{a}_{1}, \mathfrak{a}_{2}\right]$; then,

$$
\begin{aligned}
& \frac{\Omega\left(\mathfrak{a}_{1}\right)+\Omega\left(\mathfrak{a}_{2}\right)}{2}-\frac{\Gamma(\alpha+1)}{2}\left(\frac{\mathfrak{a}_{1} \mathfrak{a}_{2}}{\mathfrak{a}_{2}-\mathfrak{a}_{1}}\right)^{\alpha}\left[J_{1 / \mathfrak{a}_{1}^{-}}^{\alpha}(\Omega \circ \mathfrak{g})\left(\frac{1}{\mathfrak{a}_{2}}\right)+I_{1 / \mathfrak{a}_{2}^{+}}^{\alpha}(\Omega \circ \mathfrak{g})\left(\frac{1}{\mathfrak{a}_{1}}\right)\right] \\
& =\frac{\mathfrak{a}_{1} \mathfrak{a}_{2}\left(\mathfrak{a}_{2}-\mathfrak{a}_{1}\right)}{2} \int_{0}^{1} \frac{\left[\eta^{\alpha}-(1-\eta)^{\alpha}\right]}{\left(\eta \mathfrak{a}_{1}+(1-\eta) \mathfrak{a}_{2}\right)^{2}} \Omega^{\prime}\left(\frac{\mathfrak{a}_{1} \mathfrak{a}_{2}}{\eta \mathfrak{a}_{1}+(1-\eta) \mathfrak{a}_{2}}\right) \mathrm{d} \eta, \\
& \Omega\left(\frac{2 \mathfrak{a}_{1} \mathfrak{a}_{2}}{\mathfrak{a}_{1}+\mathfrak{a}_{2}}\right)\left[J_{1 / \mathfrak{a}_{1}^{-}}^{\alpha}(\omega \circ \mathfrak{g})\left(\frac{1}{\mathfrak{a}_{2}}\right)+J_{1 / \mathfrak{a}_{2}^{+}}^{\alpha}(\omega \circ \mathfrak{g})\left(\frac{1}{\mathfrak{a}_{1}}\right)\right]
\end{aligned}
$$

where $\mathfrak{g}(\mathfrak{i t})=1 / \mathfrak{t}$.

In [21], İşcan and Kunt represented the Hermite-Hadamard-Fejér-type inequality for harmonically convex functions in Riemann-Liouville fractional integral forms as follows.

Theorem 8 (see [21]). Let $\Omega: I \subseteq(0, \infty) \longrightarrow \mathbb{R}$ be a harmonically convex function and $\mathfrak{a}_{1}, \mathfrak{a}_{2} \in I$ with $\mathfrak{a}_{1}<\mathfrak{a}_{2}$. If $\Omega \in L\left[\mathfrak{a}_{1}, \mathfrak{a}_{2}\right]$ and $\omega:\left[\mathfrak{a}_{1}, \mathfrak{a}_{2}\right] \subseteq(0, \infty) \longrightarrow \mathbb{R}$ is nonnegative, integrable, and harmonically symmetric with respect to $2 \mathfrak{a}_{1} \mathfrak{a}_{2} / \mathfrak{a}_{1}+\mathfrak{a}_{2}$, that is, $\omega(\mathcal{x})=\omega\left(1 /\left(1 / \mathfrak{a}_{1}\right)+\left(1 / \mathfrak{a}_{2}\right)-(1 / \mathcal{\varkappa})\right)$, then$$
\begin{aligned}
& \leq\left[J_{1 / \mathfrak{a}_{1}^{-}}^{\alpha}(\Omega \omega \circ \mathfrak{g})\left(\frac{1}{\mathfrak{a}_{2}}\right)+J_{1 / \mathfrak{a}_{2}^{+}}^{\alpha}(\Omega \omega \circ \mathfrak{g})\left(\frac{1}{\mathfrak{a}_{1}}\right)\right] \\
& \leq \frac{\Omega\left(\mathfrak{a}_{1}\right)+\Omega\left(\mathfrak{a}_{2}\right)}{2}\left[J_{1 / \mathfrak{a}_{1}^{-}}^{\alpha}(\omega \circ \mathfrak{g})\left(\frac{1}{\mathfrak{a}_{2}}\right)+J_{1 / \mathfrak{a}_{2}^{+}}^{\alpha}(\omega \circ \mathfrak{g})\left(\frac{1}{\mathfrak{a}_{1}}\right)\right],
\end{aligned}
$$

where $\alpha>0 \mathfrak{g}(\mathfrak{u})=1 / \mathfrak{u}, u \in\left[1 / \mathfrak{a}_{2}, 1 / \mathfrak{a}_{1}\right]$. 
The importance of Hadamard-type inequalities is due to their roles in various fields of modern mathematics such as numerical analysis, probability, mathematical analysis, and related fields [2, 22]. Many researchers generalize and extend their studies to Hermite-Hadamard, Hermite-Hadamard-Fejér, and Pachpatte-type inequalities involving fractional integrals for various classes of convex functions (see $[20,21,23,24]$ and the references therein).

Recently (from 2020 to 2021), some new kinds of fractional treatment of Hermite-Jensen-Mercer-type inequalities for a variety of fractional integral operators were presented in [25-27]. All these results were investigated for convex functions or $s$-convex functions, and many applications to special functions like Bessel and q-digamma functions were obtained.

Since there a is massive literature about the development of fractional Mercer integral inequalities involving convex functions but still there exist many gaps to be filled for fractional integral inequalities for other classes of convex functions. Therefore, the basic aim of this paper is to present three new Hadamard-Mercer-type inequalities for harmonically convex functions using fractional integral operators with exponential kernel. We also give fractional Mercer integral inequalities for product of two harmonically convex functions. We hope that the new techniques formulated in this paper are more energizing than the accessible one.

Ahmad et al. [23] gave the definition of two new fractional integral operators with an exponential kernel.

Definition 4. Let $\Omega \in L\left(\mathfrak{a}_{1}, \mathfrak{a}_{2}\right)$. The fractional integral operators $I_{\mathfrak{a}_{1}}^{\alpha} \Omega(\mathcal{x})$ and $I_{\mathfrak{a}_{2}}^{\alpha} \Omega(\mathcal{x})$ of order $\alpha \in(0,1)$ are, respectively, defined by

$$
\begin{aligned}
& I_{\mathfrak{a}_{1}}^{\alpha} \Omega(\varkappa)=\frac{1}{\alpha} \int_{\mathfrak{a}_{1}}^{\varkappa} \exp \left(-\frac{1-\alpha}{\alpha}(\varkappa-u)\right) \Omega(u) \mathrm{d} u, \quad \varkappa>\mathfrak{a}_{1}, \\
& I_{\mathfrak{a}_{2}}^{\alpha} \Omega(\varkappa)=\frac{1}{\alpha} \int_{\varkappa}^{\mathfrak{a}_{2}} \exp \left(-\frac{1-\alpha}{\alpha}(u-\varkappa)\right) \Omega(u) \mathrm{d} u, \quad \varkappa<\mathfrak{a}_{2} .
\end{aligned}
$$

Remark 1. If $\alpha=1$, then

$$
\begin{aligned}
& \lim _{\alpha \longrightarrow 1} I_{\mathfrak{a}_{1}}^{\alpha} \Omega(\varkappa)=\int_{\mathfrak{a}_{1}}^{\varkappa} \Omega(u) \mathrm{d} u, \\
& \lim _{\alpha \longrightarrow 1} I_{\mathfrak{a}_{2}}^{\alpha} \Omega(\varkappa)=\int_{\varkappa}^{\mathfrak{a}_{2}} \Omega(u) \mathrm{d} u .
\end{aligned}
$$

For the convenience of expression, throughout the paper, we set

$$
\rho=\frac{1-\alpha}{\alpha}\left(\frac{\mathfrak{a}_{2}-\mathfrak{a}_{1}}{\mathfrak{a}_{1} \mathfrak{a}_{2}}\right) .
$$

\section{Hermite-Hadamard-Mercer-Type Inequalities for Harmonically Convex Function}

Theorem 9. Suppose that $\Omega:\left[\theta_{1}, \theta_{2}\right] \subseteq(0, \infty) \longrightarrow \mathbb{R}$ is a positive function with $0 \leq \theta_{1}<\theta_{2}$. If $\Omega$ is a harmonically convex function on $\left[\theta_{1}, \theta_{2}\right]$ and $\Omega \in L\left[\theta_{1}, \theta_{2}\right]$, then

$$
\begin{aligned}
& \Omega\left(\frac{1}{\left(1 / \theta_{1}\right)+\left(1 / \theta_{2}\right)-\left(\mathfrak{a}_{1}+\mathfrak{a}_{2} / 2 \mathfrak{a}_{1} \mathfrak{a}_{2}\right)}\right) \leq \Omega\left(\theta_{1}\right)+ \Omega\left(\theta_{2}\right)-\frac{1-\alpha}{2[1-\exp (-\rho)]}\left[I_{1 / \mathfrak{a}_{1}}^{\alpha}(\Omega \circ \mathfrak{g})\left(\frac{1}{\mathfrak{a}_{2}}\right)+I_{1 / \mathfrak{a}_{2}}^{\alpha}(\Omega \circ \mathfrak{g})\left(\frac{1}{\mathfrak{a}_{1}}\right)\right] \\
& \leq \Omega\left(\theta_{1}\right)+\Omega\left(\theta_{2}\right)-\Omega\left(\frac{2 \mathfrak{a}_{1} \mathfrak{a}_{2}}{\mathfrak{a}_{1}+\mathfrak{a}_{2}}\right), \\
& \Omega\left(\frac{1}{\left(1 / \theta_{1}\right)+\left(1 / \theta_{2}\right)-\left(\mathfrak{a}_{1}+\mathfrak{a}_{2} / 2 \mathfrak{a}_{1} \mathfrak{a}_{2}\right)}\right) \leq \frac{1-\alpha}{2[1-\exp (-\rho)]} \\
& \cdot {\left[I_{\left(\left(1 / \theta_{1}\right)+\left(1 / \theta_{2}\right)-\left(1 / \mathfrak{a}_{1}\right)\right)(\Omega \circ \mathfrak{g})\left(\frac{1}{\theta_{1}}+\frac{1}{\theta_{2}}-\frac{1}{\mathfrak{a}_{2}}\right)}^{\alpha}\right.} \\
&\left.+I_{\left(\left(1 / \theta_{1}\right)+\left(1 / \theta_{2}\right)-\left(1 / \mathfrak{a}_{2}\right)\right)}^{\alpha}(\Omega \circ \mathfrak{g})\left(\frac{1}{\theta_{1}}+\frac{1}{\theta_{2}}-\frac{1}{\mathfrak{a}_{1}}\right)\right] \\
& \leq \frac{1}{2}\left[\Omega\left(\frac{1}{\left(1 / \theta_{1}\right)+\left(1 / \theta_{2}\right)-\left(1 / \mathfrak{a}_{1}\right)}\right)+\Omega\left(\frac{1}{\left(1 / \theta_{1}\right)+\left(1 / \theta_{2}\right)-1 / \mathfrak{a}_{2}}\right)\right] \\
& \leq \Omega\left(\theta_{1}\right)+\Omega\left(\theta_{2}\right)-\frac{\Omega\left(\mathfrak{a}_{1}\right)+\Omega\left(\mathfrak{a}_{2}\right)}{2},
\end{aligned}
$$

for all $\mathfrak{a}_{1}, \mathfrak{a}_{2} \in\left[\theta_{1}, \theta_{2}\right], \alpha>0, \mathfrak{g}(\mathfrak{u t})=1 / \mathfrak{t}, u \in\left[1 / \theta_{2}, 1 / \theta_{1}\right]$ and $\rho$ is defined in (17).

$$
\begin{aligned}
\Omega\left(\frac{1}{\left(1 / \theta_{1}\right)+\left(1 / \theta_{2}\right)-\left(\overline{\mathfrak{a}_{1}}+\overline{\mathfrak{a}_{2}} / 2 \overline{\mathfrak{a}_{1} \mathfrak{a}_{1}}\right)}\right) \leq & \Omega\left(\theta_{1}\right)+\Omega\left(\theta_{2}\right) \\
& -\frac{\Omega\left(\overline{\mathfrak{a}_{1}}\right)+\Omega\left(\overline{\mathfrak{a}_{2}}\right)}{2},
\end{aligned}
$$

Proof. Using the Jensen-Mercer inequality for harmonically convex function, we have 
for all $\overline{\mathfrak{a}_{1}}, \overline{\mathfrak{a}_{2}} \in\left[\theta_{1}, \theta_{2}\right]$. By changing of the variables $\overline{\mathfrak{a}_{1}}=\mathfrak{a}_{1} \mathfrak{a}_{2} / \eta \mathfrak{a}_{1}+(1-\eta) \mathfrak{a}_{2}, \overline{\mathfrak{a}_{2}}=\mathfrak{a}_{1} \mathfrak{a}_{2} / \eta \mathfrak{a}_{2}+(1-\eta) \mathfrak{a}_{1}$ for all $\mathfrak{a}_{1}, \mathfrak{a}_{2} \in\left[\theta_{1}, \theta_{2}\right]$ and $\eta \in[0,1]$ in (20), we obtain

$$
\Omega\left(\frac{1}{\left(1 / \theta_{1}\right)+\left(1 / \theta_{2}\right)-\left(\mathfrak{a}_{1}+\mathfrak{a}_{2} / 2 \mathfrak{a}_{1} \mathfrak{a}_{2}\right)}\right) \leq \Omega\left(\theta_{1}\right)+\Omega\left(\theta_{2}\right)-\frac{1}{2}\left[\Omega\left(\frac{\mathfrak{a}_{1} \mathfrak{a}_{2}}{\eta \mathfrak{a}_{1}+(1-\eta) \mathfrak{a}_{2}}\right)+\Omega\left(\frac{\mathfrak{a}_{1} \mathfrak{a}_{2}}{\eta \mathfrak{a}_{2}+(1-\eta) \mathfrak{a}_{1}}\right)\right]
$$

Multiplying by $\exp (-\rho \eta)$ on both sides of (21) and then integrating with respect to $\eta$ over $[0,1]$, we have

$$
\begin{aligned}
& \frac{[1-\exp (-\rho)]}{\rho} \Omega\left(\frac{1}{\left(1 / \theta_{1}\right)+\left(1 / \theta_{2}\right)-\left(\mathfrak{a}_{1}+\mathfrak{a}_{2} / 2 \mathfrak{a}_{1} \mathfrak{a}_{2}\right)}\right) \\
\leq & \frac{[1-\exp (-\rho)]}{\rho}\left[\Omega\left(\mathfrak{a}_{1}\right)+\Omega\left(\mathfrak{a}_{2}\right)\right]-\frac{1}{2} \int_{0}^{1} \exp (-\rho \eta) \\
& \cdot\left[\Omega\left(\frac{\mathfrak{a}_{1} \mathfrak{a}_{2}}{\eta \mathfrak{a}_{1}+(1-\eta) \mathfrak{a}_{2}}\right)+\Omega\left(\frac{\mathfrak{a}_{1} \mathfrak{a}_{2}}{\eta \mathfrak{a}_{2}+(1-\eta) \mathfrak{a}_{1}}\right)\right] \mathrm{d} \eta \\
= & \frac{[1-\exp (-\rho)]}{\rho}\left[\Omega\left(\theta_{1}\right)+\Omega\left(\theta_{2}\right)\right]-\frac{\mathfrak{a}_{1} \mathfrak{a}_{2}}{2\left(\mathfrak{a}_{2}-\mathfrak{a}_{1}\right)}\left[\int_{1 / \mathfrak{a}_{2}}^{1 / \mathfrak{a}_{1}} \exp \left(-\frac{1-\alpha}{\alpha}\left(\frac{1}{\mathfrak{a}_{1}}-u\right)\right) \Omega\left(\frac{1}{u}\right) \mathrm{d} u\right. \\
& \left.+\int_{1 / \mathfrak{a}_{2}}^{1 / \mathfrak{a}_{1}} \exp \left(-\frac{1-\alpha}{\alpha}\left(u-\frac{1}{\mathfrak{a}_{2}}\right)\right) \Omega\left(\frac{1}{u}\right) \mathrm{d} u\right] \\
= & \frac{[1-\exp (-\rho)]}{\rho}\left[\Omega\left(\theta_{1}\right)+\Omega\left(\theta_{2}\right)\right]-\frac{\alpha \mathfrak{a}_{1} \mathfrak{a}_{2}}{2\left(\mathfrak{a}_{2}-\mathfrak{a}_{1}\right)}\left[I_{1 / \mathfrak{a}_{2}}^{\alpha}(\Omega \circ \mathfrak{g})\left(\frac{1}{\mathfrak{a}_{1}}\right)+I_{1 / \mathfrak{a}_{1}}^{\alpha}(\Omega \circ \mathfrak{g})\left(\frac{1}{\mathfrak{a}_{2}}\right)\right] .
\end{aligned}
$$

Multiplying by $\rho /[1-\exp (-\rho)]$ on both sides of above equation and putting the value of $\rho$ which is given in (17), we get

$$
\Omega\left(\frac{1}{\left(1 / \theta_{1}\right)+\left(1 / \theta_{2}\right)-\mathfrak{a}_{1}+\mathfrak{a}_{2} / 2 \mathfrak{a}_{1} \mathfrak{a}_{2}}\right) \leq \Omega\left(\theta_{1}\right)+\Omega\left(\theta_{2}\right)-\frac{1-\alpha}{2[1-\exp (-\rho)]}\left[I_{1 / \mathfrak{a}_{1}}^{\alpha}(\Omega \circ \mathfrak{g})\left(\frac{1}{\mathfrak{a}_{2}}\right)+I_{1 / \mathfrak{a}_{2}}^{\alpha}(\Omega \circ \mathfrak{g})\left(\frac{1}{\mathfrak{a}_{1}}\right)\right]
$$

Thus, the first inequality of (18) is proved. Now we prove the second inequality in (18); since $\Omega$ is a harmonically convex function, then, for $\eta \in[0,1]$, it yields

$$
\begin{aligned}
\Omega\left(\frac{2 \mathfrak{a}_{1} \mathfrak{a}_{2}}{\mathfrak{a}_{1}+\mathfrak{a}_{2}}\right)= & \Omega\left(\frac{2}{\left(\eta / \mathfrak{a}_{1}\right)+\left(1-\eta / \mathfrak{a}_{2}\right)+\left(1-\eta / \mathfrak{a}_{1}\right)+\left(\eta / \mathfrak{a}_{2}\right)}\right) \\
& \leq \frac{1}{2}\left[\Omega\left(\frac{\mathfrak{a}_{1} \mathfrak{a}_{2}}{\eta \mathfrak{a}_{2}+(1-\eta) \mathfrak{a}_{1}}\right)+\Omega\left(\frac{\mathfrak{a}_{1} \mathfrak{a}_{2}}{\eta \mathfrak{a}_{1}+(1-\eta) \mathfrak{a}_{2}}\right)\right] .
\end{aligned}
$$

Multiplying by $\exp (-\rho \eta)$ on both sides of (24) and then integrating with respect to $\eta$ over $[0,1]$, we have

$$
\frac{[1-\exp (-\rho)]}{\rho} \Omega\left(\frac{2 \mathfrak{a}_{1} \mathfrak{a}_{2}}{\mathfrak{a}_{1}+\mathfrak{a}_{2}}\right)
$$

$$
\begin{aligned}
\leq & \frac{1}{2}\left[\int_{0}^{1} \exp (-\rho \eta) \Omega\left(\frac{\mathfrak{a}_{1} \mathfrak{a}_{2}}{\eta \mathfrak{a}_{2}+(1-\eta) \mathfrak{a}_{1}}\right)\right. \\
& \left.+\Omega\left(\frac{\mathfrak{a}_{1} \mathfrak{a}_{2}}{\eta \mathfrak{a}_{1}+(1-\eta) \mathfrak{a}_{2}}\right) \mathrm{d} \eta\right] \\
= & \frac{\alpha \mathfrak{a}_{1} \mathfrak{a}_{2}}{2\left(\mathfrak{a}_{2}-\mathfrak{a}_{1}\right)}\left[I_{1 / \mathfrak{a}_{1}}^{\alpha}(\Omega \circ \mathfrak{g})\left(\frac{1}{\mathfrak{a}_{2}}\right)+I_{1 / \mathfrak{a}_{2}}^{\alpha}(\Omega \circ \mathfrak{g})\left(\frac{1}{\mathfrak{a}_{1}}\right)\right] .
\end{aligned}
$$


Then,

$$
\begin{aligned}
-\Omega\left(\frac{2 \mathfrak{a}_{1} \mathfrak{a}_{2}}{\mathfrak{a}_{1}+\mathfrak{a}_{2}}\right) \geq & -\frac{1-\alpha}{2[1-\exp (-\rho)]} \\
& \cdot\left[I_{1 / \mathfrak{a}_{1}}^{\alpha}(\Omega \circ \mathfrak{g})\left(\frac{1}{\mathfrak{a}_{2}}\right)+I_{1 / \mathfrak{a}_{2}}^{\alpha}(\Omega \circ \mathfrak{g})\left(\frac{1}{\mathfrak{a}_{1}}\right)\right] .
\end{aligned}
$$

Adding $\Omega\left(\mathfrak{a}_{1}\right)+\Omega\left(\mathfrak{a}_{2}\right)$ to both sides of (26), we find the second inequality of (18).

Now, we prove inequality (19). Since $\Omega$ is a harmonically convex function, then, we have that for any $\mathfrak{a}_{1}, \mathfrak{a}_{2} \in\left[\theta_{1}, \theta_{2}\right]$,

$$
\begin{aligned}
\Omega\left(\frac{2 \mathfrak{a}_{1} \mathfrak{a}_{2}}{\mathfrak{a}_{1}+\mathfrak{a}_{2}}\right) & =\Omega\left(\frac{1}{1 / 2\left(\left(\eta / \mathfrak{a}_{1}\right)+\left(1-\eta / \mathfrak{a}_{2}\right)+\left(1-\eta / \mathfrak{a}_{1}\right)+\left(\eta / \mathfrak{a}_{2}\right)\right)}\right) \\
& \leq \frac{1}{2}\left[\Omega\left(\frac{1}{\left(\eta / \mathfrak{a}_{1}\right)+\left(1-\eta / \mathfrak{a}_{2}\right)}\right)+\Omega\left(\frac{1}{\left(1-\eta / \mathfrak{a}_{1}\right)+\left(\eta / \mathfrak{a}_{2}\right)}\right)\right] \\
& \leq \frac{\Omega\left(\mathfrak{a}_{1}\right)+\Omega\left(\mathfrak{a}_{2}\right)}{2} .
\end{aligned}
$$

Replacing $\mathfrak{a}_{1}$ and $\mathfrak{a}_{2}$ by $1 /\left(1 / \theta_{1}\right)+\left(1 / \theta_{2}\right)-\left(1 / \mathfrak{a}_{1}\right)$ and $1 /\left(1 / \theta_{1}\right)+\left(1 / \theta_{2}\right)-\left(1 / \mathfrak{a}_{2}\right)$, respectively, in $(27)$, we get

$$
\begin{aligned}
\Omega\left(\frac{1}{\left(1 / \theta_{1}\right)+\left(1 / \theta_{2}\right)-\left(\mathfrak{a}_{1}+\mathfrak{a}_{2} / 2 \mathfrak{a}_{1} \mathfrak{a}_{2}\right)}\right) & \leq \frac{1}{2}\left[\Omega\left(\frac{1}{\left(1 / \theta_{1}\right)+\left(1 / \theta_{2}\right)-\left(\eta / \mathfrak{a}_{1}+1-\eta / \mathfrak{a}_{2}\right)}\right)+\Omega\left(\frac{1}{\left(1 / \theta_{1}\right)+\left(1 / \theta_{2}\right)-\left(1-\eta / \mathfrak{a}_{1}+\eta / \mathfrak{a}_{2}\right)}\right)\right] \\
& \leq \frac{1}{2}\left[\Omega\left(\frac{1}{\left(1 / \theta_{1}\right)+\left(1 / \theta_{2}\right)-\left(1 / \mathfrak{a}_{1}\right)}\right)+\Omega\left(\frac{1}{\left(1 / \theta_{1}\right)+\left(1 / \theta_{2}\right)-\left(1 / \mathfrak{a}_{2}\right)}\right)\right] .
\end{aligned}
$$

Multiplying by exp $(-\rho \eta)$ on both sides of (28) and then integrating with respect to $\eta$ over $[0,1]$, we obtain

$$
\begin{aligned}
& \frac{[1-\exp (-\rho)]}{\rho} \Omega\left(\frac{1}{\left(1 / \theta_{1}\right)+\left(1 / \theta_{2}\right)-\left(\mathfrak{a}_{1}+\mathfrak{a}_{2} / 2 \mathfrak{a}_{1} \mathfrak{a}_{2}\right)}\right) \\
& \leq \frac{1}{2}\left[\int_{0}^{1} \exp (-\rho \eta) \Omega\left(\frac{1}{\left(1 / \theta_{1}\right)+\left(1 / \theta_{2}\right)-\left(\eta / \mathfrak{a}_{1}+1-\eta / \mathfrak{a}_{2}\right)}\right) \mathrm{d} \eta\right. \\
& \left.+\int_{0}^{1} \exp (-\rho \eta) \Omega\left(\frac{1}{\left(1 / \theta_{1}\right)+\left(1 / \theta_{2}\right)-\left(1-\eta / \mathfrak{a}_{1}+\eta / \mathfrak{a}_{2}\right)}\right) \mathrm{d} \eta\right] \\
& \leq \frac{[1-\exp (-\rho)]}{2 \rho}\left[\Omega\left(\frac{1}{\left(1 / \theta_{1}\right)+\left(1 / \theta_{2}\right)-\left(1 / \mathfrak{a}_{1}\right)}\right)+\Omega\left(\frac{1}{\left(1 / \theta_{1}\right)+\left(1 / \theta_{2}\right)-\left(1 / \mathfrak{a}_{2}\right)}\right)\right]
\end{aligned}
$$

It is obvious that 


$$
\begin{aligned}
& \frac{1}{2}\left[\int_{0}^{1} \exp (-\rho \eta) \Omega\left(\frac{1}{1 / \theta_{1}+1 / \theta_{2}-\left(\left(\eta / \mathfrak{a}_{1}\right)+\left(1-\eta / \mathfrak{a}_{2}\right)\right)}\right) \mathrm{d} \eta+\int_{0}^{1} \exp (-\rho \eta) \Omega\left(\frac{1}{1 / \theta_{1}+1 / \theta_{2}-\left(1-\eta / \mathfrak{a}_{1}+\eta / \mathfrak{a}_{2}\right)}\right) \mathrm{d} \eta\right] \\
& =\frac{\mathfrak{a}_{1} \mathfrak{a}_{2}}{2\left(\mathfrak{a}_{2}-\mathfrak{a}_{1}\right)}\left[\int_{\left(1 / \theta_{1}\right)+\left(1 / \theta_{2}\right)-\left(1 / \mathfrak{a}_{1}\right)}^{\left(1 / \theta_{1}\right)+\left(1 / \theta_{2}\right)-\left(1 / \mathfrak{a}_{2}\right)} \exp \left(-\frac{1-\alpha}{\alpha}\left(\left(\frac{1}{\theta_{1}}+\frac{1}{\theta_{2}}-\frac{1}{\mathfrak{a}_{2}}\right)-u\right)\right) \Omega\left(\frac{1}{u}\right) \mathrm{d} u\right. \\
& \left.\quad+\int_{\left(1 / \theta_{1}\right)+\left(1 / \theta_{2}\right)-\left(1 / \mathfrak{a}_{1}\right)}^{\left(1 / \theta_{1}\right)+\left(1 / \theta_{2}\right)-\left(1 / \mathfrak{a}_{2}\right)} \exp \left(-\frac{1-\alpha}{\alpha}\left(u-\left(\frac{1}{\theta_{1}}+\frac{1}{\theta_{2}}-\frac{1}{\mathfrak{a}_{1}}\right)\right)\right) \Omega\left(\frac{1}{u}\right) \mathrm{d} u\right] \\
& =\frac{\alpha \mathfrak{a}_{1} \mathfrak{a}_{2}}{2\left(\mathfrak{a}_{2}-\mathfrak{a}_{1}\right)}\left[I_{\left(\left(1 / \theta_{1}\right)+\left(1 / \theta_{2}\right)-\left(1 / \mathfrak{a}_{1}\right)\right)}^{\alpha}(\Omega \circ \mathfrak{g})\left(\frac{1}{\theta_{1}}+\frac{1}{\theta_{2}}-\frac{1}{\mathfrak{a}_{2}}\right)+I_{\left(\left(1 / \theta_{1}\right)+\left(1 / \theta_{2}\right)-\left(1 / \mathfrak{a}_{2}\right)\right)}^{\alpha}(\Omega \circ \mathfrak{g})\left(\frac{1}{\theta_{1}}+\frac{1}{\theta_{2}}-\frac{1}{\mathfrak{a}_{1}}\right)\right]
\end{aligned}
$$

Using the Jensen-Mercer inequality for harmonically convex function, we conclude that

$$
\begin{aligned}
& \Omega\left(\frac{1}{\left(1 / \theta_{1}\right)+\left(1 / \theta_{2}\right)-\left(\mathfrak{a}_{1}+\mathfrak{a}_{2} / 2 \mathfrak{a}_{1} \mathfrak{a}_{2}\right)}\right) \\
& \quad \leq \frac{1-\alpha}{2[1-\exp (-\rho)]}\left[I_{\left(\left(1 / \theta_{1}\right)+\left(1 / \theta_{2}\right)-\left(1 / \mathfrak{a}_{1}\right)\right)}^{\alpha}(\Omega \circ \mathfrak{g})\left(\frac{1}{\theta_{1}}+\frac{1}{\theta_{2}}-\frac{1}{\mathfrak{a}_{2}}\right)+I_{\left(\left(1 / \theta_{1}\right)+\left(1 / \theta_{2}\right)-\left(1 / \mathfrak{a}_{2}\right)\right)}^{\alpha}(\Omega \circ \mathfrak{g})\left(\frac{1}{\theta_{1}}+\frac{1}{\theta_{2}}-\frac{1}{\mathfrak{a}_{1}}\right)\right] \\
& \quad \leq \frac{1}{2}\left[\Omega\left(\frac{1}{1 / \theta_{1}+1 / \theta_{1}-1 / \mathfrak{a}_{1}}\right)+\Omega\left(\frac{1}{1 / \theta_{1}+1 / \theta_{1}-1 / \mathfrak{a}_{2}}\right)\right] \\
& \quad \leq \Omega\left(\theta_{1}\right)+\Omega\left(\theta_{2}\right)-\frac{\Omega\left(\mathfrak{a}_{1}\right)+\Omega\left(\mathfrak{a}_{2}\right)}{2}
\end{aligned}
$$

So, inequality (19) is proved.

$$
\lim _{\alpha \longrightarrow 1} \frac{1-\alpha}{2[1-\exp (-\rho)]}=\frac{\mathfrak{a}_{1} \mathfrak{a}_{2}}{2\left(\mathfrak{a}_{2}-\mathfrak{a}_{1}\right)} .
$$

Remark 2. If we take $\mathfrak{a}_{1}=\theta_{1}$ and $\mathfrak{a}_{2}=\theta_{2}$ in Theorem 9 , then we have Theorem 2.1 in [24].

Under the assumptions of Theorem 9 with $\alpha=1$, one has

Remark 3. For $\alpha \longrightarrow 1$, we have

$$
\begin{aligned}
\Omega\left(\frac{1}{\left(1 / \theta_{1}\right)+\left(1 / \theta_{2}\right)-\left(\mathfrak{a}_{1}+\mathfrak{a}_{2} / 2 \mathfrak{a}_{1} \mathfrak{a}_{2}\right)}\right) & \leq \Omega\left(\theta_{1}\right)+\Omega\left(\theta_{2}\right)-\int_{0}^{1} \Omega\left(\frac{\mathfrak{a}_{1} \mathfrak{a}_{2}}{\eta \mathfrak{a}_{1}+(1-\eta) \mathfrak{a}_{2}}\right) \mathrm{d} \eta \\
& \leq \Omega\left(\theta_{1}\right)+\Omega\left(\theta_{2}\right)-\Omega\left(\frac{2 \mathfrak{a}_{1} \mathfrak{a}_{2}}{\mathfrak{a}_{1}+\mathfrak{a}_{2}}\right), \\
\Omega\left(\frac{1}{\left(1 / \theta_{1}\right)+\left(1 / \theta_{2}\right)-\left(\mathfrak{a}_{1}+\mathfrak{a}_{2} / 2 \mathfrak{a}_{1} \mathfrak{a}_{2}\right)}\right) & \leq \frac{\mathfrak{a}_{1} \mathfrak{a}_{2}}{\mathfrak{a}_{2}-\mathfrak{a}_{1}} \int_{\mathfrak{a}_{1}}^{\mathfrak{a}_{2}} \frac{1}{\eta^{2}} \Omega\left(\frac{1}{\left(1 / \theta_{1}\right)+\left(1 / \theta_{2}\right)-(1 / \eta)}\right) \mathrm{d} \eta \\
& \leq \Omega\left(\theta_{1}\right)+\Omega\left(\theta_{2}\right)-\frac{\Omega\left(\mathfrak{a}_{1}\right)+\Omega\left(\mathfrak{a}_{2}\right)}{2},
\end{aligned}
$$

for all $\mathfrak{a}_{1}, \mathfrak{a}_{2} \in\left[\theta_{1}, \theta_{2}\right]$. Inequalities (33) and (34) were proved by Baloch et al. in [28, Theorem 3.5] and [29, Theorem 2.1].
Remark 4. If $\alpha \longrightarrow 1, \mathfrak{a}_{1}=\theta_{1}$, and $\mathfrak{a}_{2}=\theta_{2}$ in Theorem 9, then we have Hermite-Hadamard inequality (6) for harmonically convex function which was proved by İşcan in [11]. 
Lemma 3. Let $\Omega:\left[\theta_{1}, \theta_{2}\right] \subseteq(0, \infty) \longrightarrow \mathbb{R}$ be a differentiable function on $\left(\theta_{1}, \theta_{2}\right)$ with $\theta_{1}<\theta_{2}$. If $\Omega^{\prime} \in L\left[\theta_{1}, \theta_{2}\right]$, then

$$
\begin{aligned}
& \frac{1}{2}\left[\Omega\left(\frac{1}{\left(1 / \theta_{1}\right)+\left(1 / \theta_{2}\right)-\left(1 / \mathfrak{a}_{1}\right)}\right)+\Omega\left(\frac{1}{\left(1 / \theta_{1}\right)+\left(1 / \theta_{2}\right)-\left(1 / \mathfrak{a}_{2}\right)}\right)\right]-\frac{1-\alpha}{2[1-\exp (-\rho)]}
\end{aligned}
$$

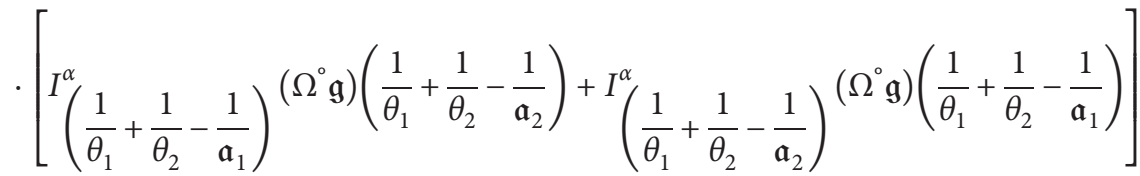

$$
\begin{aligned}
& =\frac{\mathfrak{a}_{2}-\mathfrak{a}_{1}}{2 \mathfrak{a}_{1} \mathfrak{a}_{2}[1-\exp (-\rho)]}\left[\int_{0}^{1} \frac{\exp (-\rho(1-\eta))}{\left(1 / \theta_{1}\right)+\left(1 / \theta_{2}\right)-\left(\left(\eta / \mathfrak{a}_{1}\right)+\left(1-\eta / \mathfrak{a}_{2}\right)\right)^{2}} \Omega^{\prime}\left(\frac{1}{\left(1 / \theta_{1}\right)+\left(1 / \theta_{2}\right)-\left(\left(\eta / \mathfrak{a}_{1}\right)+\left(1-\eta / \mathfrak{a}_{2}\right)\right)}\right) \mathrm{d} \eta\right. \\
& \left.-\int_{0}^{1} \frac{\exp (-\rho \eta)}{\left(\left(1 / \theta_{1}\right)+\left(1 / \theta_{2}\right)-\left(\left(\eta / \mathfrak{a}_{1}\right)+\left(1-\eta / \mathfrak{a}_{2}\right)\right)\right)^{2}} \Omega^{\prime}\left(\frac{1}{\left(1 / \theta_{1}\right)+\left(1 / \theta_{2}\right)-\left(\left(\eta / \mathfrak{a}_{1}\right)+\left(1-\eta / \mathfrak{a}_{2}\right)\right)}\right) \mathrm{d} \eta\right] \text {, }
\end{aligned}
$$

for all $\mathfrak{a}_{1}, \mathfrak{a}_{2} \in\left[\theta_{1}, \theta_{2}\right], \quad \alpha>0, \quad$ and $\mathfrak{g}(\mathfrak{u})=1 / \mathfrak{t}, \quad$ Proof. Let $A_{\eta}=1 / \theta_{1}+1 / \theta_{2}-\left(\eta / \mathfrak{a}_{1}+1-\eta / \mathfrak{a}_{2}\right)$. It suffices to $u \in\left[1 / \theta_{2}, 1 / \theta_{1}\right]$. note that

$$
\begin{aligned}
\frac{1}{2}\left[\Omega\left(\frac{1}{\left(1 / \theta_{1}\right)+\left(1 / \theta_{2}\right)-\left(1 / \mathfrak{a}_{1}\right)}\right)+\Omega\left(\frac{1}{\left(1 / \theta_{1}\right)+\left(1 / \theta_{2}\right)-\left(1 / \mathfrak{a}_{2}\right)}\right)\right]-\frac{1-\alpha}{2[1-\exp (-\rho)]} \\
\quad \cdot\left[I_{\left(1 / \theta_{1}+1 / \theta_{1}-1 / \mathfrak{a}_{1}\right)}^{\alpha}(\Omega \circ \mathfrak{g})\left(\frac{1}{\theta_{1}}+\frac{1}{\theta_{2}}-\frac{1}{\mathfrak{a}_{2}}\right)+I_{\left(1 / \theta_{1}+1 / \theta_{1}-1 / \mathfrak{a}_{2}\right)}^{\alpha}(\Omega \circ \mathfrak{g})\left(\frac{1}{\theta_{1}}+\frac{1}{\theta_{2}}-\frac{1}{\mathfrak{a}_{1}}\right)\right] \\
=\frac{\mathfrak{a}_{2}-\mathfrak{a}_{1}}{2 \mathfrak{a}_{1} \mathfrak{a}_{2}[1-\exp (-\rho)]}\left[\int_{0}^{1} \frac{\exp (-\rho(1-\eta))}{A_{\eta}^{2}} \Omega^{\prime}\left(\frac{1}{\left(1 / \theta_{1}\right)+\left(1 / \theta_{2}\right)-\left(\left(\eta / \mathfrak{a}_{1}\right)+\left(1-\eta / \mathfrak{a}_{2}\right)\right)}\right) \mathrm{d} \eta\right. \\
\left.\quad-\int_{0}^{1} \frac{\exp (-\rho \eta)}{A_{\eta}^{2}} \Omega^{\prime}\left(\frac{1}{\left(1 / \theta_{1}\right)+\left(1 / \theta_{2}\right)-\left(\left(\eta / \mathfrak{a}_{1}\right)+\left(1-\eta / \mathfrak{a}_{2}\right)\right)}\right) \mathrm{d} \eta\right]=I_{1}-I_{2} .
\end{aligned}
$$

By integrating by part, we have

$$
\begin{aligned}
& I_{1}=\frac{1}{2[1-\exp (-\rho)]}\left[\left.\exp (-\rho(1-\eta)) \Omega\left(\frac{1}{A_{\eta}}\right)\right|_{0} ^{1}-\rho \int_{0}^{1} \exp (-\rho \eta) \Omega\left(\frac{1}{A_{\eta}}\right) \mathrm{d} \eta\right] \\
& =\frac{1}{2[1-\exp (-\rho)]}\left[\Omega\left(\frac{1}{\left(1 / \theta_{1}\right)+\left(1 / \theta_{2}\right)-\left(1 / \mathfrak{a}_{1}\right)}\right)-\exp (-\rho) \Omega\left(\frac{1}{\left(1 / \theta_{1}\right)+\left(1 / \theta_{2}\right)-\left(1 / \mathfrak{a}_{2}\right)}\right)\right. \\
& \left.-\rho\left(\frac{\mathfrak{a}_{1} \mathfrak{a}_{2}}{\mathfrak{a}_{2}-\mathfrak{a}_{1}}\right) \int_{1 / \theta_{1}+1 / \theta_{2}-1 / \mathfrak{a}_{1}}^{1 / \theta_{1}+1 / \theta_{2}-1 / \mathfrak{a}_{2}} \exp \left(-\frac{1-\alpha}{\alpha}\left(u-\left(\frac{1}{\theta_{1}}+\frac{1}{\theta_{2}}-\frac{1}{\mathfrak{a}_{1}}\right)\right)\right) \Omega\left(\frac{1}{u}\right) \mathrm{d} u\right] \\
& =\frac{1}{2[1-\exp (-\rho)]}\left[\Omega\left(\frac{1}{\left(1 / \theta_{1}\right)+\left(1 / \theta_{2}\right)-\left(1 / \mathfrak{a}_{1}\right)}\right)-\exp (-\rho) \Omega\left(\frac{1}{\left(1 / \theta_{1}\right)+\left(1 / \theta_{2}\right)-\left(1 / \mathfrak{a}_{2}\right)}\right)\right. \\
& \left.-\rho\left(\frac{\alpha \mathfrak{a}_{1} \mathfrak{a}_{2}}{\mathfrak{a}_{2}-\mathfrak{a}_{1}}\right) I_{\left(1 / \theta_{1}+1 / \theta_{2}-1 / \mathfrak{a}_{2}\right)}^{\alpha}(\Omega \circ \mathfrak{g})\left(\frac{1}{\theta_{1}}+\frac{1}{\theta_{2}}-\frac{1}{\mathfrak{a}_{1}}\right)\right],
\end{aligned}
$$


and similarly we get

$$
\begin{aligned}
I_{2}= & \frac{1}{2[1-\exp (-\rho)]}\left[\left.\exp (-\rho \eta) \Omega\left(\frac{1}{A_{\eta}}\right)\right|_{0} ^{1}-(-\rho) \int_{0}^{1} \exp (-\rho \eta) \Omega\left(\frac{1}{A_{\eta}}\right) \mathrm{d} \eta\right] \\
= & \frac{1}{2[1-\exp (-\rho)]}\left[\exp (-\rho) \Omega\left(\frac{1}{\left(1 / \theta_{1}\right)+\left(1 / \theta_{1}\right)-\left(1 / \mathfrak{a}_{1}\right)}\right)-\Omega\left(\frac{1}{\left(1 / \theta_{1}\right)+\left(1 / \theta_{2}\right)-\left(1 / \mathfrak{a}_{2}\right)}\right)\right. \\
& \left.+\rho\left(\frac{\mathfrak{a}_{1} \mathfrak{a}_{2}}{\mathfrak{a}_{2}-\mathfrak{a}_{1}}\right) \int \frac{\left.1 / \theta_{1}\right)+\left(1 / \theta_{1}\right)-\left(1 / \mathfrak{a}_{2}\right)}{\left(1 / \theta_{1}\right)+\left(1 / \theta_{1}\right)-\left(1 / \mathfrak{a}_{1}\right)} \exp \left(-\frac{1-\alpha}{\alpha}\left(\left(\frac{1}{\theta_{1}}+\frac{1}{\theta_{2}}-\frac{1}{\mathfrak{a}_{2}}\right)-u\right)\right) \Omega\left(\frac{1}{u}\right) \mathrm{d} u\right] \\
= & \frac{1}{2[1-\exp (-\rho)]}\left[\exp (-\rho) \Omega\left(\frac{1}{\left(1 / \theta_{1}\right)+\left(1 / \theta_{2}\right)-\left(1 / \mathfrak{a}_{1}\right)}\right)-\Omega\left(\frac{1}{\left(1 / \theta_{1}\right)+\left(1 / \theta_{2}\right)-\left(1 / \mathfrak{a}_{2}\right)}\right)\right. \\
& \left.+\rho\left(\frac{\alpha \mathfrak{a}_{1} \mathfrak{a}_{2}}{\mathfrak{a}_{2}-\mathfrak{a}_{1}}\right) I_{\left(1 / \theta_{1}\right)+\left(1 / \theta_{1}\right)-\left(1 / \mathfrak{a}_{1}\right)}^{\alpha}(\Omega \circ \mathfrak{g})\left(\frac{1}{\theta_{1}}+\frac{1}{\theta_{2}}-\frac{1}{\mathfrak{a}_{2}}\right)\right] .
\end{aligned}
$$

Using (37) and (40) in (36), we get equality (35).

Remark 5. From Lemma 2 with $\alpha \longrightarrow 1, \mathfrak{a}_{1}=\theta_{1}$, and $\mathfrak{a}_{2}=\theta_{2}$, we indeed have Lemma 1 which was proved by Íşcan in [11].
Theorem 10. If $\Omega: I=\left[\theta_{1}, \theta_{2}\right] \subseteq(0, \infty) \longrightarrow \mathbb{R}$ is a differentiable function on $\left(\theta_{1}, \theta_{2}\right)$ with $\theta_{1}<\theta_{2}$ and $\Omega^{\prime} \in L\left[\theta_{1}, \theta_{2}\right]$. If $\left|\Omega^{\prime}\right|$ is a harmonically convex on $\left[\theta_{1}, \theta_{2}\right]$, then

$$
\begin{aligned}
& \mid \frac{1}{2}\left[\Omega\left(\frac{1}{\left(1 / \theta_{1}\right)+\left(1 / \theta_{2}\right)-\left(1 / \mathfrak{a}_{1}\right)}\right)+\Omega\left(\frac{1}{\left(1 / \theta_{1}\right)+\left(1 / \theta_{2}\right)-\left(1 / \mathfrak{a}_{2}\right)}\right)\right]-\frac{1-\alpha}{2[1-\exp (-\rho)]}\left[I_{\left(1 / \theta_{1}+1 / \theta_{2}-1 / \mathfrak{a}_{1}\right)}^{\alpha}(\Omega \circ \mathfrak{g})\left(\frac{1}{\theta_{1}}+\frac{1}{\theta_{2}}-\frac{1}{\mathfrak{a}_{2}}\right)\right. \\
& \left.\quad+I_{\left(1 / \theta_{1}+1 / \theta_{2}-1 / \mathfrak{a}_{2}\right)}^{\alpha}(\Omega \circ \mathfrak{g})\left(\frac{1}{\theta_{1}}+\frac{1}{\theta_{2}}-\frac{1}{\mathfrak{a}_{1}}\right)\right] \mid \\
& \leq \frac{\mathfrak{a}_{2}-\mathfrak{a}_{1}}{2 \mathfrak{a}_{1} \mathfrak{a}_{2}[1-\exp (-\rho)]} \int_{0}^{1 / 2}[\exp (-\rho \eta)-\exp (-\rho(1-\eta))]\left[\left(\frac{\eta}{\mu_{1}^{2}}+\frac{1-\eta}{\mu_{2}^{2}}\right)\left|\Omega^{\prime}\left(\frac{1}{\left(1 / \theta_{1}\right)+\left(1 / \theta_{2}\right)-\left(1 / \mathfrak{a}_{1}\right)}\right)\right|\right. \\
& \left.\quad+\left(\frac{1-\eta}{\mu_{1}^{2}}+\frac{\eta}{\mu_{2}^{2}}\right)\left|\Omega^{\prime}\left(\frac{1}{\left(1 / \theta_{1}\right)+\left(1 / \theta_{2}\right)-\left(1 / \mathfrak{a}_{2}\right)}\right)\right|\right] d \eta,
\end{aligned}
$$

where

$$
\begin{aligned}
& \mu_{1}=\frac{1}{\theta_{1}}+\frac{1}{\theta_{2}}-\left(\frac{\eta}{\mathfrak{a}_{1}}+\frac{1-\eta}{\mathfrak{a}_{2}}\right), \\
& \mu_{2}=\frac{1}{\theta_{1}}+\frac{1}{\theta_{2}}-\left(\frac{1-\eta}{\mathfrak{a}_{1}}+\frac{\eta}{\mathfrak{a}_{2}}\right),
\end{aligned}
$$

for all $\mathfrak{a}_{1}, \mathfrak{a}_{2} \in\left[\theta_{1}, \theta_{2}\right], \quad \alpha>0, \quad$ and $\quad \mathfrak{g}(\mathfrak{u})=1 / \mathfrak{l}$, $u \in\left[1 / \theta_{2}, 1 / \theta_{1}\right]$.

Proof. Since $\left|\Omega^{\prime}\right|$ is a harmonically convex on $\left[\theta_{1}, \theta_{2}\right]$, using Lemma 2, we can obtain 


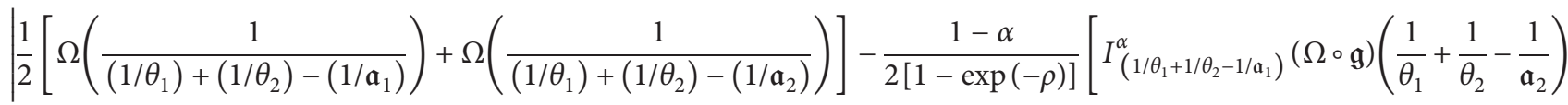

$$
\begin{aligned}
& \left.+I_{\left(1 / \theta_{1}+1 / \theta_{2}-1 / \mathfrak{a}_{2}\right)}^{\alpha}(\Omega \circ \mathfrak{g})\left(\frac{1}{\theta_{1}}+\frac{1}{\theta_{2}}-\frac{1}{\mathfrak{a}_{1}}\right)\right] \mid \\
& \leq \frac{\mathfrak{a}_{2}-\mathfrak{a}_{1}}{2 \mathfrak{a}_{1} \mathfrak{a}_{2}[1-\exp (-\rho)]} \int_{0}^{1} \frac{|\exp (-\rho \eta)-\exp (-\rho(1-\eta))|}{\left(\left(1 / \theta_{1}\right)+\left(1 / \theta_{2}\right)-\left(\left(\eta / \mathfrak{a}_{1}\right)+\left(1-\eta / \mathfrak{a}_{2}\right)\right)\right)^{2}}\left|\Omega^{\prime}\left(\frac{1}{\left(1 / \theta_{1}\right)+\left(1 / \theta_{2}\right)-\left(\left(\eta / \mathfrak{a}_{1}\right)+\left(1-\eta / \mathfrak{a}_{2}\right)\right)}\right)\right| \mathrm{d} \eta \\
& \leq \frac{\mathfrak{a}_{2}-\mathfrak{a}_{1}}{2 \mathfrak{a}_{1} \mathfrak{a}_{2}[1-\exp (-\rho)]}\left[\int _ { 0 } ^ { 1 / 2 } \frac { [ \operatorname { e x p } ( - \rho \eta ) - \operatorname { e x p } ( - \rho ( 1 - \eta ) ) ] } { ( ( 1 / \theta _ { 1 } ) + ( 1 / \theta _ { 2 } ) - ( ( \eta / \mathfrak { a } _ { 1 } ) + ( 1 - \eta / \mathfrak { a } _ { 2 } ) ) ) ^ { 2 } } \left(\eta\left|\Omega^{\prime}\left(\frac{1}{\left(1 / \theta_{1}\right)+\left(1 / \theta_{2}\right)-\left(1 / \mathfrak{a}_{1}\right)}\right)\right|\right.\right. \\
& \left.+(1-\eta)\left|\Omega^{\prime}\left(\frac{1}{\left(1 / \theta_{1}\right)+\left(1 / \theta_{2}\right)-\left(1 / \mathfrak{a}_{2}\right)}\right)\right|\right) \mathrm{d} \eta+\int_{1 / 2}^{1} \frac{[\exp (-\rho(1-\eta))-\exp (-\rho \eta)]}{\left(\left(1 / \theta_{1}\right)+\left(1 / \theta_{2}\right)-\left(\left(\eta / \mathfrak{a}_{1}\right)+\left(1-\eta / \mathfrak{a}_{2}\right)\right)\right)^{2}} \\
& \cdot\left(\eta\left|\Omega^{\prime}\left(\frac{1}{\left(1 / \theta_{1}\right)+\left(1 / \theta_{2}\right)-\left(1 / \mathfrak{a}_{1}\right)}\right)\right|\right. \\
& \left.\left.+(1-\eta)\left|\Omega^{\prime}\left(\frac{1}{\left(1 / \theta_{1}\right)+\left(1 / \theta_{2}\right)-\left(1 / \mathfrak{a}_{2}\right)}\right)\right|\right) \mathrm{d} \eta\right] \leq \frac{\mathfrak{a}_{2}-\mathfrak{a}_{1}}{2 \mathfrak{a}_{1} \mathfrak{a}_{2}[1-\exp (-\rho)]} \\
& \cdot\left[\int _ { 0 } ^ { 1 / 2 } \frac { [ \operatorname { e x p } ( - \rho \eta ) - \operatorname { e x p } ( - \rho ( 1 - \eta ) ) ] } { ( ( 1 / \theta _ { 1 } ) + ( 1 / \theta _ { 2 } ) - ( ( \eta / \mathfrak { a } _ { 1 } ) + ( 1 - \eta / \mathfrak { a } _ { 2 } ) ) ) ^ { 2 } } \left(\eta\left|\Omega^{\prime}\left(\frac{1}{\left(1 / \theta_{1}\right)+\left(1 / \theta_{2}\right)-\left(1 / \mathfrak{a}_{1}\right)}\right)\right|\right.\right. \\
& \left.+(1-\eta)\left|\Omega^{\prime}\left(\frac{1}{\left(1 / \theta_{1}\right)+\left(1 / \theta_{2}\right)-\left(1 / \mathfrak{a}_{2}\right)}\right)\right|\right) \mathrm{d} \eta+\int_{0}^{1 / 2} \frac{[\exp (-\rho \eta)-\exp (-\rho(1-\eta))]}{\left(\left(1 / \theta_{1}\right)+\left(1 / \theta_{2}\right)-\left(\left(1-\eta / \mathfrak{a}_{1}\right)+\left(\eta / \mathfrak{a}_{2}\right)\right)\right)^{2}} \\
& \cdot\left((1-\eta)\left|\Omega^{\prime}\left(\frac{1}{\left(1 / \theta_{1}\right)+\left(1 / \theta_{2}\right)-\left(1 / \mathfrak{a}_{1}\right)}\right)\right|\right. \\
& \left.\left.+\eta\left|\Omega^{\prime}\left(\frac{1}{\left(1 / \theta_{1}\right)+\left(1 / \theta_{2}\right)-\left(1 / \mathfrak{a}_{2}\right)}\right)\right|\right) \mathrm{d} \eta\right] \\
& =\frac{\mathfrak{a}_{2}-\mathfrak{a}_{1}}{2 \mathfrak{a}_{1} \mathfrak{a}_{2}[1-\exp (-\rho)]} \int_{0}^{1 / 2}[\exp (-\rho \eta)-\exp (-\rho(1-\eta))] \\
& \cdot\left[\left(\frac{\eta}{\mu_{1}^{2}}+\frac{1-\eta}{\mu_{2}^{2}}\right)\left|\Omega^{\prime}\left(\frac{1}{\left(1 / \theta_{1}\right)+\left(1 / \theta_{2}\right)-\left(1 / \mathfrak{a}_{1}\right)}\right)+\left(\frac{1-\eta}{\mu_{1}^{2}}+\frac{\eta}{\mu_{2}^{2}}\right)\right| \Omega^{\prime}\left(\frac{1}{\left(1 / \theta_{1}\right)+\left(1 / \theta_{2}\right)-\left(1 / \mathfrak{a}_{2}\right)}\right) \mid\right] \mathrm{d} \eta \mid .
\end{aligned}
$$

This completes the proof.

Remark 6. For $\alpha \longrightarrow 1$, we have

$$
\begin{aligned}
\lim _{\alpha \longrightarrow 1} \frac{1-\alpha}{2[1-\exp (-\rho)]} & =\frac{\mathfrak{a}_{1} \mathfrak{a}_{2}}{2\left(\mathfrak{a}_{2}-\mathfrak{a}_{1}\right)}, \\
\lim _{\alpha \longrightarrow 1} \frac{\exp (-\rho \eta)-\exp (-\rho(1-\eta))}{2[1-\exp (-\rho)]} & =\frac{1-2 \eta}{2} .
\end{aligned}
$$

If $\alpha \longrightarrow 1, \mathfrak{a}_{1}=\theta_{1}$, and $\mathfrak{a}_{2}=\theta_{2}$ in Theorem 10 , then we get Theorem 2.6 which was proved by İşcan in [11].

\section{Fejér-Hadamard-Mercer-Type Inequality for Harmonically Convex Function}

Theorem 11. If $\Omega: I \subseteq(0, \infty) \longrightarrow \mathbb{R}$ is a harmonically convex function for $\theta_{1}, \theta_{2} \in I$ with $\theta_{1}<\theta_{2}$. such that If $\Omega \in L\left[\theta_{1}, \theta_{2}\right]$ and $\omega:\left[\theta_{1}, \theta_{2}\right] \subseteq(0, \infty) \longrightarrow \mathbb{R}$ is nonnegative, 
integrable, and harmonically symmetric with respect to $2 \theta_{1} \theta_{2} / \theta_{1}+\theta_{2}$, that is, $\omega(\varkappa)=\omega\left(1 /\left(1 / \theta_{1}\right)+\left(1 / \theta_{2}\right)-(1 / \varkappa)\right)$, then

$$
\begin{aligned}
& \Omega\left(\frac{1}{\left(1 / \theta_{1}\right)+\left(1 / \theta_{2}\right)-\left(\mathfrak{a}_{1}+\mathfrak{a}_{2} / 2 \mathfrak{a}_{1} \mathfrak{a}_{2}\right)}\right)\left[I_{\left(1 / \theta_{1}+1 / \theta_{2}-1 / \mathfrak{a}_{1}\right)}^{\alpha}(\omega \circ \mathfrak{g})\left(\frac{1}{\theta_{1}}+\frac{1}{\theta_{2}}-\frac{1}{\mathfrak{a}_{2}}\right)+I_{\left(1 / \theta_{1}+1 / \theta_{2}-1 / \mathfrak{a}_{2}\right)}^{\alpha}(\omega \circ \mathfrak{g})\left(\frac{1}{\theta_{1}}+\frac{1}{\theta_{2}}-\frac{1}{\mathfrak{a}_{1}}\right)\right] \\
& \leq\left[I_{\left(1 / \theta_{1}+1 / \theta_{2}-1 / \mathfrak{a}_{1}\right)}^{\alpha}(\omega \circ \mathfrak{g})\left(\frac{1}{\theta_{1}}+\frac{1}{\theta_{2}}-\frac{1}{\mathfrak{a}_{2}}\right)+I_{\left(1 / \theta_{1}+1 / \theta_{2}-1 / \mathfrak{a}_{2}\right)}^{\alpha}(\Omega \omega \circ \mathfrak{g})\left(\frac{1}{\theta_{1}}+\frac{1}{\theta_{2}}-\frac{1}{\mathfrak{a}_{1}}\right)\right] \\
& \leq \frac{1}{2}\left[\Omega\left(\frac{1}{\left(1 / \theta_{1}\right)+\left(1 / \theta_{2}\right)-\left(1 / \mathfrak{a}_{1}\right)}\right)+\Omega\left(\frac{1}{\left(1 / \theta_{1}\right)+\left(1 / \theta_{2}\right)-\left(1 / \mathfrak{a}_{2}\right)}\right)\right]\left[I_{\left(1 / \theta_{1}+1 / \theta_{2}-1 / \mathfrak{a}_{1}\right)}^{\alpha}(\omega \circ \mathfrak{g})\left(\frac{1}{\theta_{1}}+\frac{1}{\theta_{2}}-\frac{1}{\mathfrak{a}_{2}}\right)\right. \\
& \left.\quad+I_{\left(1 / \theta_{1}+1 / \theta_{2}-1 / \mathfrak{a}_{2}\right)}^{\alpha}(\omega \circ \mathfrak{g})\left(\frac{1}{\theta_{1}}+\frac{1}{\theta_{2}}-\frac{1}{\mathfrak{a}_{1}}\right)\right]
\end{aligned}
$$

for all $\mathfrak{a}_{1}, \mathfrak{a}_{2} \in\left[\theta_{1}, \theta_{2}\right], \quad \alpha>0, \quad$ and $\mathfrak{g}(\mathfrak{u})=1 / \mathfrak{u}$, Proof. If $\Omega$ is a harmonically convex function on $\left[\theta_{1}, \theta_{2}\right]$, $u \in\left[1 / \theta_{2}, 1 / \theta_{1}\right]$. then for all $\mathfrak{a}_{1}, \mathfrak{a}_{2} \in\left[\theta_{1}, \theta_{2}\right]$,

$$
\begin{aligned}
\Omega\left(\frac{2 \mathfrak{a}_{1} \mathfrak{a}_{2}}{\mathfrak{a}_{1}+\mathfrak{a}_{2}}\right) & =\Omega\left(\frac{1}{1 / 2\left(\left(\eta / \mathfrak{a}_{1}\right)+\left(1-\eta / \mathfrak{a}_{2}\right)+\left(1-\eta / \mathfrak{a}_{1}\right)+\eta / \mathfrak{a}_{2}\right)}\right) \\
& \leq \frac{1}{2}\left[\Omega\left(\frac{1}{\left(\eta / \mathfrak{a}_{1}\right)+\left(1-\eta / \mathfrak{a}_{2}\right)}\right)+\Omega\left(\frac{1}{\left(1-\eta / \mathfrak{a}_{1}\right)+\left(\eta / \mathfrak{a}_{2}\right)}\right)\right] \leq \frac{\Omega\left(\mathfrak{a}_{1}\right)+\Omega\left(\mathfrak{a}_{2}\right)}{2} .
\end{aligned}
$$

Replacing $\mathfrak{a}_{1}$ and $\mathfrak{a}_{2}$ by $1 /\left(1 / \theta_{1}\right)+\left(1 / \theta_{2}\right)-\left(1 / \mathfrak{a}_{1}\right)$ and $1 /\left(1 / \theta_{1}\right)+\left(1 / \theta_{2}\right)-\left(1 / \mathfrak{a}_{2}\right)$, respectively, we get

$$
\begin{aligned}
\Omega\left(\frac{1}{\left(1 / \theta_{1}\right)+\left(1 / \theta_{2}\right)-\left(\mathfrak{a}_{1}+\mathfrak{a}_{2} / 2 \mathfrak{a}_{1} \mathfrak{a}_{2}\right)}\right) \leq & \frac{1}{2}\left[\Omega\left(\frac{1}{\left(1 / \theta_{1}\right)+\left(1 / \theta_{2}\right)-\left(\left(\eta / \mathfrak{a}_{1}\right)+\left(1-\eta / \mathfrak{a}_{2}\right)\right)}\right)\right. \\
& \left.+\Omega\left(\frac{1}{\left(1 / \theta_{1}\right)+\left(1 / \theta_{2}\right)-\left(\left(1-\eta / \mathfrak{a}_{1}\right)+\left(\eta / \mathfrak{a}_{2}\right)\right)}\right)\right] \\
\leq & {\left[\Omega\left(\frac{1}{\left(1 / \theta_{1}\right)+\left(1 / \theta_{2}\right)-\left(1 / \mathfrak{a}_{1}\right)}\right)+\Omega\left(\frac{1}{\left(1 / \theta_{1}\right)+\left(1 / \theta_{2}\right)-\left(1 / \mathfrak{a}_{2}\right)}\right)\right] }
\end{aligned}
$$

Multiplying by

$$
\exp (-\rho \eta) \omega\left(\frac{1}{\left(1 / \theta_{1}\right)+\left(1 / \theta_{2}\right)-\left(\left(1-\eta / \mathfrak{a}_{1}\right)+\left(\eta / \mathfrak{a}_{2}\right)\right)}\right)
$$

on both sides of (46) and then integrating with respect to $\eta$ over $[0,1]$, we obtain 
12

Mathematical Problems in Engineering

$$
\begin{aligned}
& 2 \Omega\left(\frac{1}{\left(1 / \theta_{1}\right)+\left(1 / \theta_{2}\right)-\left(\mathfrak{a}_{1}+\mathfrak{a}_{2} / 2 \mathfrak{a}_{1} \mathfrak{a}_{2}\right)}\right) \int_{0}^{1} \exp (-\rho \eta) \omega\left(\frac{1}{\left(1 / \theta_{1}\right)+\left(1 / \theta_{2}\right)-\left(\left(1-\eta / \mathfrak{a}_{1}\right)+\left(\eta / \mathfrak{a}_{2}\right)\right)}\right) \mathrm{d} \eta \\
& \quad \leq \int_{0}^{1} \exp (-\rho \eta) \Omega\left(\frac{1}{\left(1 / \theta_{1}\right)+\left(1 / \theta_{2}\right)-\left(\left(\eta / \mathfrak{a}_{1}\right)+\left(1-\eta / \mathfrak{a}_{2}\right)\right)}\right) \omega\left(\frac{1}{\left(1 / \theta_{1}\right)+\left(1 / \theta_{2}\right)-\left(\left(1-\eta / \mathfrak{a}_{1}\right)+\left(\eta / \mathfrak{a}_{2}\right)\right)}\right) \mathrm{d} \eta \\
& \quad+\int_{0}^{1} \exp (-\rho \eta) \Omega\left(\frac{1}{\left(1 / \theta_{1}\right)+\left(1 / \theta_{2}\right)-\left(\left(1-\eta / \mathfrak{a}_{1}\right)+\left(\eta / \mathfrak{a}_{2}\right)\right)}\right) \omega\left(\frac{1}{\left(1 / \theta_{1}\right)+\left(1 / \theta_{2}\right)-\left(\left(1-\eta / \mathfrak{a}_{1}\right)+\left(\eta / \mathfrak{a}_{2}\right)\right)}\right) \mathrm{d} \eta \\
& \quad \leq\left[\Omega\left(\frac{1}{\left(1 / \theta_{1}\right)+\left(1 / \theta_{2}\right)-\left(1 / \mathfrak{a}_{1}\right)}\right)+\Omega\left(\frac{1}{\left(1 / \theta_{1}\right)+\left(1 / \theta_{2}\right)-\left(1 / \mathfrak{a}_{2}\right)}\right)\right] \int_{0}^{1} \exp (-\rho \eta) \omega\left(\frac{1}{\left(1 / \theta_{1}\right)+\left(1 / \theta_{2}\right)-\left(\left(1-\eta / \mathfrak{a}_{1}\right)+\left(\eta / \mathfrak{a}_{2}\right)\right)}\right) \mathrm{d} \eta,
\end{aligned}
$$

where

$$
\begin{aligned}
& \int_{0}^{1} \exp (-\rho \eta) \Omega\left(\frac{1}{\left(1 / \theta_{1}\right)+\left(1 / \theta_{2}\right)-\left(\left(\eta / \mathfrak{a}_{1}\right)+\left(1-\eta / \mathfrak{a}_{2}\right)\right)}\right) \omega\left(\frac{1}{\left(1 / \theta_{1}\right)+\left(1 / \theta_{2}\right)-\left(\left(1-\eta / \mathfrak{a}_{1}\right)+\left(\eta / \mathfrak{a}_{2}\right)\right)}\right) \mathrm{d} \eta \\
& +\int_{0}^{1} \exp (-\rho \eta) \Omega\left(\frac{1}{\left(1 / \theta_{1}\right)+\left(1 / \theta_{2}\right)-\left(\left(1-\eta / \mathfrak{a}_{1}\right)+\left(\eta / \mathfrak{a}_{2}\right)\right)}\right) \omega\left(\frac{1}{\left(1 / \theta_{1}\right)+\left(1 / \theta_{2}\right)-\left(\left(1-\eta / \mathfrak{a}_{1}\right)+\left(\eta / \mathfrak{a}_{2}\right)\right)}\right) \mathrm{d} \eta \\
& =\frac{\mathfrak{a}_{1} \mathfrak{a}_{2}}{\mathfrak{a}_{2}-\mathfrak{a}_{1}}\left[\int_{1 / \theta_{1}+1 / \theta_{2}-1 / \mathfrak{a}_{1}}^{1 / \theta_{1}+1 / \theta_{2}-1 / \mathfrak{a}_{2}} \exp \left(-\frac{1-\alpha}{\alpha}\left(u-\left(\frac{1}{\theta_{1}}+\frac{1}{\theta_{2}}-\frac{1}{\mathfrak{a}_{1}}\right)\right)\right) \Omega\left(\frac{1}{\left(\left(1 / \theta_{1}\right)+\left(1 / \theta_{2}\right)-\left(1 / \mathfrak{a}_{1}\right)\right)+\left(\left(1 / \theta_{1}\right)+\left(1 / \theta_{2}\right)-\left(1 / \mathfrak{a}_{2}\right)\right)-u}\right)\right. \\
& \left.\omega\left(\frac{1}{u}\right) \mathrm{d} u+\int_{1 / \theta_{1}+1 / \theta_{2}-1 / \mathfrak{a}_{1}}^{1 / \theta_{1}+1 / \theta_{2}-1 / \mathfrak{a}_{2}} \exp \left(-\frac{1-\alpha}{\alpha}\left(u-\left(\frac{1}{\theta_{1}}+\frac{1}{\theta_{2}}-\frac{1}{\mathfrak{a}_{1}}\right)\right)\right) \Omega\left(\frac{1}{u}\right) \omega\left(\frac{1}{u}\right) \mathrm{d} u\right] \\
& =\frac{\mathfrak{a}_{1} \mathfrak{a}_{2}}{\mathfrak{a}_{2}-\mathfrak{a}_{1}}\left[\int_{1 / \theta_{1}+1 / \theta_{2}-1 / \mathfrak{a}_{1}}^{1 / \theta_{1}+1 / \theta_{2}-1 / \mathfrak{a}_{2}} \exp \left(-\frac{1-\alpha}{\alpha}\left(\left(\frac{1}{\theta_{1}}+\frac{1}{\theta_{2}}-\frac{1}{\mathfrak{a}_{2}}\right)-u\right)\right) \Omega\left(\frac{1}{u}\right) \omega\right. \\
& \cdot\left(\frac{1}{\left(\left(1 / \theta_{1}\right)+\left(1 / \theta_{2}\right)-\left(1 / \mathfrak{a}_{1}\right)\right)+\left(\left(1 / \theta_{1}\right)+\left(1 / \theta_{2}\right)-\left(1 / \mathfrak{a}_{2}\right)\right)-u}\right) \mathrm{d} u \\
& \left.+\int_{1 / \theta_{1}+1 / \theta_{2}-1 / \mathfrak{a}_{1}}^{1 / \theta_{1}+1 / \theta_{2}-1 / \mathfrak{a}_{2}} \exp \left(-\frac{1-\alpha}{\alpha}\left(u-\left(\left(1 / \theta_{1}\right)+\left(1 / \theta_{2}\right)-\left(1 / \mathfrak{a}_{1}\right)\right)\right)\right) \Omega\left(\frac{1}{u}\right) \omega\left(\frac{1}{u}\right) \mathrm{d} u\right] \\
& =\frac{\alpha \mathfrak{a}_{1} \mathfrak{a}_{2}}{\mathfrak{a}_{2}-\mathfrak{a}_{1}}\left[I_{\left(1 / \theta_{1}+1 / \theta_{2}-1 / \mathfrak{a}_{1}\right)}^{\alpha}(\Omega \omega \circ \mathfrak{g})\left(\frac{1}{\theta_{1}}+\frac{1}{\theta_{2}}-\frac{1}{\mathfrak{a}_{2}}\right)+I_{\left(1 / \theta_{1}+1 / \theta_{2}-1 / \mathfrak{a}_{2}\right)}^{\alpha}(\Omega \omega \circ \mathfrak{g})\left(\frac{1}{\theta_{1}}+\frac{1}{\theta_{2}}-\frac{1}{\mathfrak{a}_{1}}\right)\right] .
\end{aligned}
$$

That is,

$$
\begin{aligned}
& 2 \Omega\left(\frac{1}{\left(1 / \theta_{1}\right)+\left(1 / \theta_{2}\right)-\left(\mathfrak{a}_{1}+\mathfrak{a}_{2} / 2 \mathfrak{a}_{1} \mathfrak{a}_{2}\right)}\right) \int_{0}^{1} \exp (-\rho \eta) \omega\left(\frac{1}{\left(1 / \theta_{1}\right)+\left(1 / \theta_{2}\right)-\left(\left(1-\eta / \mathfrak{a}_{1}\right)+\left(\eta / \mathfrak{a}_{2}\right)\right)}\right) \mathrm{d} \eta \\
& \quad \leq \frac{\alpha \mathfrak{a}_{1} \mathfrak{a}_{2}}{\mathfrak{a}_{2}-\mathfrak{a}_{1}}\left[I_{\left(1 / \theta_{1}+1 / \theta_{2}-1 / \mathfrak{a}_{1}\right)}^{\alpha}(\Omega \omega \circ \mathfrak{g})\left(\frac{1}{\theta_{1}}+\frac{1}{\theta_{2}}-\frac{1}{\mathfrak{a}_{2}}\right)+I_{\left(1 / \theta_{1}+1 / \theta_{2}-1 / \mathfrak{a}_{2}\right)}^{\alpha}(\Omega \omega \circ \mathfrak{g})\left(\frac{1}{\theta_{1}}+\frac{1}{\theta_{2}}-\frac{1}{\mathfrak{a}_{1}}\right)\right] \\
& \quad \leq\left[\Omega\left(\frac{1}{\left(1 / \theta_{1}\right)+\left(1 / \theta_{2}\right)-\left(1 / \mathfrak{a}_{1}\right)}\right)+\Omega\left(\frac{1}{\left(1 / \theta_{1}\right)+\left(1 / \theta_{2}\right)-\left(1 / \mathfrak{a}_{2}\right)}\right)\right] \int_{0}^{1} \exp (-\rho \eta) \\
& \quad \cdot \omega\left(\frac{1}{\left(1 / \theta_{1}\right)+\left(1 / \theta_{2}\right)-\left(\left(1-\eta / \mathfrak{a}_{1}\right)+\left(\eta / \mathfrak{a}_{2}\right)\right)}\right) \mathrm{d} \eta .
\end{aligned}
$$


Since $\omega$ is symmetric with respect to $2 \theta_{1} \theta_{2} / \theta_{1}+\theta_{2}$, we have

$$
\begin{aligned}
& I_{\left(1 / \theta_{1}+1 / \theta_{2}-1 / \mathfrak{a}_{2}\right)}^{\alpha}(\omega \circ \mathfrak{g})\left(\frac{1}{\theta_{1}}+\frac{1}{\theta_{2}}-\frac{1}{\mathfrak{a}_{1}}\right) \\
& \quad=I_{\left(1 / \theta_{1}+1 / \theta_{2}-1 / \mathfrak{a}_{1}\right)}^{\alpha}(\omega \circ \mathfrak{g})\left(\frac{1}{\theta_{1}}+\frac{1}{\theta_{2}}-\frac{1}{\mathfrak{a}_{2}}\right) \\
& \quad=\frac{1}{2}\left[I_{\left(1 / \theta_{1}+1 / \theta_{2}-1 / \mathfrak{a}_{1}\right)}^{\alpha}(\omega \circ \mathfrak{g})\left(\frac{1}{\theta_{1}}+\frac{1}{\theta_{2}}-\frac{1}{\mathfrak{a}_{2}}\right)+I_{\left(1 / \theta_{1}+1 / \theta_{2}-1 / \mathfrak{a}_{2}\right)}^{\alpha}(\omega \circ \mathfrak{g})\left(\frac{1}{\theta_{1}}+\frac{1}{\theta_{2}}-\frac{1}{\mathfrak{a}_{1}}\right)\right] .
\end{aligned}
$$

Therefore, we have

$$
\begin{aligned}
& \Omega\left(\frac{1}{\left(1 / \theta_{1}\right)+\left(1 / \theta_{2}\right)-\left(\mathfrak{a}_{1}+\mathfrak{a}_{2} / 2 \mathfrak{a}_{1} \mathfrak{a}_{2}\right)}\right)\left[I_{\left(1 / \theta_{1}+1 / \theta_{2}-1 / \mathfrak{a}_{1}\right)}^{\alpha}(\omega \circ \mathfrak{g})\left(\frac{1}{\theta_{1}}+\frac{1}{\theta_{2}}-\frac{1}{\mathfrak{a}_{2}}\right)+I_{\left(1 / \theta_{1}+1 / \theta_{2}-1 / \mathfrak{a}_{2}\right)}^{\alpha}(\omega \circ \mathfrak{g})\left(\frac{1}{\theta_{1}}+\frac{1}{\theta_{2}}-\frac{1}{\mathfrak{a}_{1}}\right)\right] \\
& \leq\left[I_{\left(1 / \theta_{1}+1 / \theta_{2}-1 / \mathfrak{a}_{1}\right)}^{\alpha}(\Omega \omega \circ \mathfrak{g})\left(\frac{1}{\theta_{1}}+\frac{1}{\theta_{2}}-\frac{1}{\mathfrak{a}_{2}}\right)+I_{\left(1 / \theta_{1}+1 / \theta_{2}-1 / \mathfrak{a}_{2}\right)}^{\alpha}(\Omega \omega \circ \mathfrak{g})\left(\frac{1}{\theta_{1}}+\frac{1}{\theta_{2}}-\frac{1}{\mathfrak{a}_{1}}\right)\right] \\
& \leq \frac{1}{2}\left[\Omega\left(\frac{1}{\left(1 / \theta_{1}\right)+\left(1 / \theta_{1}\right)-\left(1 / \mathfrak{a}_{1}\right)}\right)+\Omega\left(\frac{1}{\left(1 / \theta_{1}\right)+\left(1 / \theta_{1}\right)-\left(1 / \mathfrak{a}_{2}\right)}\right)\right]\left[I_{\left(1 / \theta_{1}+1 / \theta_{2}-1 / \mathfrak{a}_{1}\right)}^{\alpha}(\omega \circ \mathfrak{g})\left(\frac{1}{\theta_{1}}+\frac{1}{\theta_{2}}-\frac{1}{\mathfrak{a}_{2}}\right)\right. \\
& \left.+I_{\left(1 / \theta_{1}+1 / \theta_{2}-1 / \mathfrak{a}_{2}\right)}^{\alpha}(\omega \circ \mathfrak{g})\left(\frac{1}{\theta_{1}}+\frac{1}{\theta_{2}}-\frac{1}{\mathfrak{a}_{1}}\right)\right] \text {. }
\end{aligned}
$$

Thus, the proof of Theorem 11 is complete.

Remark 7. If we take $\mathfrak{a}_{1}=\theta_{1}$ and $\mathfrak{a}_{2}=\theta_{2}$ in Theorem 11, we will get Theorem 3.2 in [24].
Remark 8. Under the assumptions of Theorem 11 with $\alpha=1$, we have

$$
\begin{aligned}
& \Omega\left(\frac{1}{\left(1 / \theta_{1}\right)+\left(1 / \theta_{2}\right)-\left(\mathfrak{a}_{1}+\mathfrak{a}_{2} / 2 \mathfrak{a}_{1} \mathfrak{a}_{2}\right)}\right) \int_{\left(1 / \theta_{1}+1 / \theta_{2}-1 / \mathfrak{a}_{1}\right)}^{\left(1 / \theta_{1}+1 / \theta_{2}-1 / \mathfrak{a}_{2}\right)} \frac{\omega(\varkappa)}{\varkappa^{2}} \mathrm{~d} \varkappa \\
& \quad \leq \int_{\left(1 / \theta_{1}+1 / \theta_{2}-1 / \mathfrak{a}_{1}\right)}^{\left(1 / \theta_{1}+1 / \theta_{2}-1 / \mathfrak{a}_{2}\right)} \frac{\Omega(\varkappa)}{\varkappa^{2}} \omega(\varkappa) d \varkappa \\
& \quad \leq \frac{1}{2}\left[\Omega\left(\frac{1}{\left(1 / \theta_{1}\right)+\left(1 / \theta_{2}\right)-\left(1 / \mathfrak{a}_{1}\right)}\right)+\Omega\left(\frac{1}{\left(1 / \theta_{1}\right)+\left(1 / \theta_{2}\right)-\left(1 / \mathfrak{a}_{2}\right)}\right)\right] \int_{\left(1 / \theta_{1}+1 / \theta_{2}-1 / \mathfrak{a}_{1}\right)}^{\left(1 / \theta_{1}+1 / \theta_{2}-1 / \mathfrak{a}_{2}\right)} \frac{\omega(\varkappa)}{\varkappa^{2}} \mathrm{~d} \varkappa .
\end{aligned}
$$


Remark 9. If $\alpha=1, \mathfrak{a}_{1}=\theta_{1}$, and $\mathfrak{a}_{2}=\theta_{2}$ in Theorem 11, then we can get Hermite-Hadamard-Fejér inequality (8) for harmonically convex function which was proved by Chen and $\mathrm{Wu}$ in [12].

\section{Pachpatte-Mercer-Type Inequality for Harmonically Convex Function}

Theorem 12. Let $\Omega, \omega:\left[\theta_{1}, \theta_{2}\right] \subseteq(0, \infty) \longrightarrow \mathbb{R}$ be functions such that $\Omega, \omega, \Omega \omega \in L\left[\theta_{1}, \theta_{2}\right]$. If $\Omega$ and $\omega$ are harmonically convex on $\left[\theta_{1}, \theta_{2}\right]$, then

$$
\begin{aligned}
& \frac{\alpha \mathfrak{a}_{1} \mathfrak{a}_{2}}{2\left(\mathfrak{a}_{2}-\mathfrak{a}_{1}\right)}\left[I_{\left(\left(1 / \theta_{1}+1 / \theta_{2}-1 / \mathfrak{a}_{1}\right)\right)}^{\alpha}(\Omega \omega \circ \mathfrak{g})\left(\frac{1}{\theta_{1}}+\frac{1}{\theta_{2}}-\frac{1}{\mathfrak{a}_{2}}\right)+I_{\left(1 / \theta_{1}+1 / \theta_{2}-1 / \mathfrak{a}_{2}\right)}^{\alpha}(\Omega \omega \circ \mathfrak{g})\left(\frac{1}{\theta_{1}}+\frac{1}{\theta_{2}}-\frac{1}{\mathfrak{a}_{1}}\right)\right] \\
& \quad \leq \frac{\rho^{2}-2 \rho+4-\left(\rho^{2}+2 \rho+4\right) \exp (-\rho)}{2 \rho^{3}} M\left(\theta_{1}, \theta_{2}, \mathfrak{a}_{1}, \mathfrak{a}_{2}\right)+\frac{\rho-2+\exp (-\rho)(\rho+2)}{\rho^{3}} N\left(\theta_{1}, \theta_{2}, \mathfrak{a}_{1}, \mathfrak{a}_{2}\right), \\
& 2 \Omega\left(\frac{1}{\left(1 / \theta_{1}\right)+\left(1 / \theta_{2}\right)-\left(\mathfrak{a}_{1}+\mathfrak{a}_{2} / 2 \mathfrak{a}_{1} \mathfrak{a}_{2}\right)}\right) \omega\left(\frac{1}{\left(1 / \theta_{1}\right)+\left(1 / \theta_{2}\right)-\left(\mathfrak{a}_{1}+\mathfrak{a}_{2} / 2 \mathfrak{a}_{1} \mathfrak{a}_{2}\right)}\right) \\
& \quad \leq \frac{1-\alpha}{2[1-\exp (-\rho)]}\left[I_{\left(1 / \theta_{1}+1 / \theta_{2}-1 / \mathfrak{a}_{1}\right)}^{\alpha}(\Omega \omega \circ \mathfrak{g})\left(\frac{1}{\theta_{1}}+\frac{1}{\theta_{2}}-\frac{1}{\mathfrak{a}_{2}}\right)+I_{\left(1 / \theta_{1}+1 / \theta_{2}-1 / \mathfrak{a}_{2}\right)}^{\alpha}(\Omega \omega \circ \mathfrak{g})\left(\frac{1}{\theta_{1}}+\frac{1}{\theta_{2}}-\frac{1}{\mathfrak{a}_{1}}\right)\right] \\
& \quad+\frac{\rho-2+\exp (-\rho)(\rho+2)}{\rho^{2}[1-\exp (-\rho)]} M\left(\theta_{1}, \theta_{2}, \mathfrak{a}_{1}, \mathfrak{a}_{2}\right)+\frac{\rho^{2}-2 \rho+4-\left(\rho^{2}+2 \rho+4\right) \exp (-\rho)}{2 \rho^{2}[1-\exp (-\rho)]} N\left(\theta_{1}, \theta_{2}, \mathfrak{a}_{1}, \mathfrak{a}_{2}\right),
\end{aligned}
$$

where

$$
\begin{aligned}
M\left(\theta_{1}, \theta_{2}, \mathfrak{a}_{1}, \mathfrak{a}_{2}\right)= & \Omega\left(\frac{1}{\left(1 / \theta_{1}\right)+\left(1 / \theta_{2}\right)-\left(1 / \mathfrak{a}_{1}\right)}\right) \omega\left(\frac{1}{\left(1 / \theta_{1}\right)+\left(1 / \theta_{2}\right)-\left(1 / \mathfrak{a}_{1}\right)}\right) \\
& +\Omega\left(\frac{1}{\left(1 / \theta_{1}\right)+\left(1 / \theta_{2}\right)-\left(1 / \mathfrak{a}_{2}\right)}\right) \omega\left(\frac{1}{\left(1 / \theta_{1}\right)+\left(1 / \theta_{2}\right)-\left(1 / \mathfrak{a}_{2}\right)}\right), \\
N\left(\theta_{1}, \theta_{2}, \mathfrak{a}_{1}, \mathfrak{a}_{2}\right)= & \Omega\left(\frac{1}{\left(1 / \theta_{1}\right)+\left(1 / \theta_{2}\right)-\left(1 / \mathfrak{a}_{1}\right)}\right) \omega\left(\frac{1}{\left(1 / \theta_{1}\right)+\left(1 / \theta_{2}\right)-\left(1 / \mathfrak{a}_{2}\right)}\right) \\
& +\Omega\left(\frac{1}{\left(1 / \theta_{1}\right)+\left(1 / \theta_{2}\right)-\left(1 / \mathfrak{a}_{2}\right)}\right) \omega\left(\frac{1}{\left(1 / \theta_{1}\right)+\left(1 / \theta_{2}\right)-\left(1 / \mathfrak{a}_{1}\right)}\right),
\end{aligned}
$$

for all $\mathfrak{a}_{1}, \mathfrak{a}_{2} \in\left[\theta_{1}, \theta_{2}\right], \quad \alpha>0, \quad$ and $\quad \mathfrak{g}(\mathfrak{u})=1 / \mathfrak{t}$, $u \in\left[1 / \theta_{2}, 1 / \theta_{1}\right]$.

Proof. Since $\Omega$ and $\omega$ are harmonically convex on $\left[\theta_{1}, \theta_{2}\right]$, then for all $\eta \in[0,1]$, we have

$$
\begin{aligned}
& \Omega\left(\frac{1}{\left(1 / \theta_{1}\right)+\left(1 / \theta_{2}\right)-\left(\left(\eta / \mathfrak{a}_{1}\right)+\left(1-\eta / \mathfrak{a}_{2}\right)\right)}\right) \omega\left(\frac{1}{\left(1 / \theta_{1}\right)+\left(1 / \theta_{2}\right)-\left(\left(\eta / \mathfrak{a}_{1}\right)+\left(1-\eta / \mathfrak{a}_{2}\right)\right)}\right) \\
& \leq \eta^{2} \Omega\left(\frac{1}{\left(1 / \theta_{1}\right)+\left(1 / \theta_{2}\right)-\left(1 / \mathfrak{a}_{1}\right)}\right) \omega\left(\frac{1}{\left(1 / \theta_{1}\right)+\left(1 / \theta_{2}\right)-\left(1 / \mathfrak{a}_{1}\right)}\right) \\
& \quad+(1-\eta)^{2} \Omega\left(\frac{1}{\left(1 / \theta_{1}\right)+\left(1 / \theta_{2}\right)-\left(1 / \mathfrak{a}_{2}\right)}\right) \omega\left(\frac{1}{\left(1 / \theta_{1}\right)+\left(1 / \theta_{2}\right)-\left(1 / \mathfrak{a}_{2}\right)}\right)
\end{aligned}
$$




$$
\begin{aligned}
&+\eta(1-\eta)\left[\Omega\left(\frac{1}{\left(1 / \theta_{1}\right)+\left(1 / \theta_{2}\right)-\left(1 / \mathfrak{a}_{1}\right)}\right) \omega\left(\frac{1}{\left(1 / \theta_{1}\right)+\left(1 / \theta_{2}\right)-\left(1 / \mathfrak{a}_{2}\right)}\right)\right. \\
&\left.+\Omega\left(\frac{1}{\left(1 / \theta_{1}\right)+\left(1 / \theta_{2}\right)-\left(1 / \mathfrak{a}_{2}\right)}\right) \omega\left(\frac{1}{\left(1 / \theta_{1}\right)+\left(1 / \theta_{2}\right)-\left(1 / \mathfrak{a}_{1}\right)}\right)\right], \\
& \Omega\left(\frac{1}{\left(1 / \theta_{1}\right)+\left(1 / \theta_{2}\right)-\left(\left(1-\eta / \mathfrak{a}_{1}\right)+\left(\eta / \mathfrak{a}_{2}\right)\right)}\right) \omega\left(\frac{1}{\left(1 / \theta_{1}\right)+\left(1 / \theta_{2}\right)-\left(\left(1-\eta / \mathfrak{a}_{1}\right)+\left(\eta / \mathfrak{a}_{2}\right)\right)}\right) \\
& \leq \eta^{2} \Omega\left(\frac{1}{\left(1 / \theta_{1}\right)+\left(1 / \theta_{2}\right)-\left(1 / \mathfrak{a}_{2}\right)}\right) \omega\left(\frac{1}{\left(1 / \theta_{1}\right)+\left(1 / \theta_{2}\right)-\left(1 / \mathfrak{a}_{2}\right)}\right) \\
&+(1-\eta)^{2} \Omega\left(\frac{1}{\left(1 / \theta_{1}\right)+\left(1 / \theta_{2}\right)-\left(1 / \mathfrak{a}_{1}\right)}\right) \omega\left(\frac{1}{\left(1 / \theta_{1}\right)+\left(1 / \theta_{2}\right)-\left(1 / \mathfrak{a}_{1}\right)}\right) \\
&+\eta(1-\eta)\left[\Omega\left(\frac{1}{\left(1 / \theta_{1}\right)+\left(1 / \theta_{2}\right)-\left(1 / \mathfrak{a}_{1}\right)}\right) \omega\left(\frac{1}{\left(1 / \theta_{1}\right)+\left(1 / \theta_{2}\right)-\left(1 / \mathfrak{a}_{2}\right)}\right)\right. \\
&\left.+\Omega\left(\frac{1}{\left(1 / \theta_{1}\right)+\left(1 / \theta_{2}\right)-\left(1 / \mathfrak{a}_{2}\right)}\right) \omega\left(\frac{1}{\left(1 / \theta_{1}\right)+\left(1 / \theta_{2}\right)-\left(1 / \mathfrak{a}_{2}\right)}\right)\right] .
\end{aligned}
$$

Adding these inequalities, we have

$$
\begin{aligned}
& \Omega\left(\frac{1}{\left(1 / \theta_{1}\right)+\left(1 / \theta_{2}\right)-\left(\left(\eta / \mathfrak{a}_{1}\right)+\left(1-\eta / \mathfrak{a}_{2}\right)\right)}\right) \omega\left(\frac{1}{\left(1 / \theta_{1}\right)+\left(1 / \theta_{2}\right)-\left(\left(\eta / \mathfrak{a}_{1}\right)+\left(1-\eta / \mathfrak{a}_{2}\right)\right)}\right) \\
& \quad+\Omega\left(\frac{1}{\left(1 / \theta_{1}\right)+\left(1 / \theta_{2}\right)-\left(\left(1-\eta / \mathfrak{a}_{1}\right)+\left(\eta / \mathfrak{a}_{2}\right)\right)}\right) \omega\left(\frac{1}{\left(1 / \theta_{1}\right)+\left(1 / \theta_{2}\right)-\left(\left(1-\eta / \mathfrak{a}_{1}\right)+\left(\eta / \mathfrak{a}_{2}\right)\right)}\right) \\
& \quad \leq\left(2 \eta^{2}-2 \eta+1\right) M\left(\theta_{1}, \theta_{2}, \mathfrak{a}_{1}, \mathfrak{a}_{2}\right)+2 \eta(1-\eta) N\left(\theta_{1}, \theta_{2}, \mathfrak{a}_{1}, \mathfrak{a}_{2}\right) .
\end{aligned}
$$

Multiplying by exp $(-\rho \eta)$ on both sides of (58) and then integrating with respect to $\eta \in[0,1]$, we have

$$
\begin{aligned}
& \int_{0}^{1} \exp (-\rho \eta) \Omega\left(\frac{1}{\left(1 / \theta_{1}\right)+\left(1 / \theta_{2}\right)-\left(\left(\eta / \mathfrak{a}_{1}\right)+\left(1-\eta / \mathfrak{a}_{2}\right)\right)}\right) \omega\left(\frac{1}{\left(1 / \theta_{1}\right)+\left(1 / \theta_{2}\right)-\left(\left(\eta / \mathfrak{a}_{1}\right)+\left(1-\eta / \mathfrak{a}_{2}\right)\right)}\right) \mathrm{d} \eta \\
& +\int_{0}^{1} \exp (-\rho \eta) \Omega\left(\frac{1}{\left(1 / \theta_{1}\right)+\left(1 / \theta_{2}\right)-\left(\left(1-\eta / \mathfrak{a}_{1}\right)+\left(\eta / \mathfrak{a}_{2}\right)\right)}\right) \omega\left(\frac{1}{\left(1 / \theta_{1}\right)+\left(1 / \theta_{2}\right)-\left(\left(1-\eta / \mathfrak{a}_{1}\right)+\left(\eta / \mathfrak{a}_{2}\right)\right)}\right) \mathrm{d} \eta \\
& \leq M\left(\theta_{1}, \theta_{2}, \mathfrak{a}_{1}, \mathfrak{a}_{2}\right) \int_{0}^{1} \exp (-\rho \eta)\left(2 \eta^{2}-2 \eta+1\right) \mathrm{d} \eta+N\left(\theta_{1}, \theta_{2}, \mathfrak{a}_{1}, \mathfrak{a}_{2}\right) \int_{0}^{1} \exp (-\rho \eta) 2 \eta(1-\eta) \mathrm{d} \eta \\
& =\frac{\rho^{2}-2 \rho+4-\left(\rho^{2}+2 \rho+4\right) \exp (-\rho)}{\rho^{3}} M\left(\theta_{1}, \theta_{2}, \mathfrak{a}_{1}, \mathfrak{a}_{2}\right)+2\left(\frac{\rho-2+\exp (-\rho)(\rho+2)}{\rho^{3}}\right) N\left(\theta_{1}, \theta_{2}, \mathfrak{a}_{1}, \mathfrak{a}_{2}\right) .
\end{aligned}
$$

Thus, we have

$$
\begin{aligned}
& \frac{\alpha \mathfrak{a}_{1} \mathfrak{a}_{2}}{2\left(\mathfrak{a}_{2}-\mathfrak{a}_{1}\right)}\left[I_{\left(1 / \theta_{1}+1 / \theta_{2}-1 / \mathfrak{a}_{1}\right)}^{\alpha}(\Omega \omega \circ \mathfrak{g})\left(\frac{1}{\theta_{1}}+\frac{1}{\theta_{2}}-\frac{1}{\mathfrak{a}_{2}}\right)+I_{\left(1 / \theta_{1}+1 / \theta_{2}-1 / \mathfrak{a}_{2}\right)}^{\alpha}(\Omega \omega \circ \mathfrak{g})\left(\frac{1}{\theta_{1}}+\frac{1}{\theta_{2}}-\frac{1}{\mathfrak{a}_{1}}\right)\right] \\
& \leq \frac{\rho^{2}-2 \rho+4-\left(\rho^{2}+2 \rho+4\right) \exp (-\rho)}{2 \rho^{3}} M\left(\theta_{1}, \theta_{2}, \mathfrak{a}_{1}, \mathfrak{a}_{2}\right)+\frac{\rho-2+\exp (-\rho)(\rho+2)}{\rho^{3}} N\left(\theta_{1}, \theta_{2}, \mathfrak{a}_{1}, \mathfrak{a}_{2}\right) .
\end{aligned}
$$


Thus, inequality (54) is proved. Now we prove inequality (55). By using harmonic convexity of the function $\Omega$ on $\left[\theta_{1}, \theta_{2}\right]$, we have

$$
\begin{aligned}
\Omega\left(\frac{2 \mathfrak{a}_{1} \mathfrak{a}_{2}}{\mathfrak{a}_{1}+\mathfrak{a}_{2}}\right) \omega\left(\frac{2 \mathfrak{a}_{1} \mathfrak{a}_{2}}{\mathfrak{a}_{1}+\mathfrak{a}_{2}}\right)= & \Omega\left(\frac{1}{1 / 2\left(\left(\eta / \mathfrak{a}_{1}\right)+\left(1-\eta / \mathfrak{a}_{2}\right)+\left(1-\eta / \mathfrak{a}_{1}\right)+\left(\eta / \mathfrak{a}_{2}\right)\right)}\right) \\
& \cdot \omega\left(\frac{1}{1 / 2\left(\left(\eta / \mathfrak{a}_{1}\right)+\left(1-\eta / \mathfrak{a}_{2}\right)+\left(1-\eta / \mathfrak{a}_{1}\right)+\left(\eta / \mathfrak{a}_{2}\right)\right)}\right) \\
\leq & \frac{1}{2}\left[\Omega\left(\frac{1}{\left(\eta / \mathfrak{a}_{1}\right)+\left(1-\eta / \mathfrak{a}_{2}\right)}\right)+\Omega\left(\frac{1}{\left(1-\eta / \mathfrak{a}_{1}\right)+\left(\eta / \mathfrak{a}_{2}\right)}\right)\right] \\
& \cdot \frac{1}{2}\left[\omega\left(\frac{1}{\left(\eta / \mathfrak{a}_{1}\right)+\left(1-\eta / \mathfrak{a}_{2}\right)}\right)+\omega\left(\frac{1}{\left(1-\eta / \mathfrak{a}_{1}\right)+\left(\eta / \mathfrak{a}_{2}\right)}\right)\right] .
\end{aligned}
$$

Replacing $\mathfrak{a}_{1}$ and $\mathfrak{a}_{2}$ by $1 /\left(1 / \theta_{1}\right)+\left(1 / \theta_{2}\right)-\left(1 / \mathfrak{a}_{1}\right)$ and $1 /\left(1 / \theta_{1}\right)+\left(1 / \theta_{2}\right)-\left(1 / \mathfrak{a}_{2}\right)$, respectively, we get

$$
\begin{aligned}
\Omega( & \left.\frac{1}{\left(1 / \theta_{1}\right)+\left(1 / \theta_{2}\right)-\left(\mathfrak{a}_{1}+\mathfrak{a}_{2} / 2 \mathfrak{a}_{1} \mathfrak{a}_{2}\right)}\right) \omega\left(\frac{1}{\left(1 / \theta_{1}\right)+\left(1 / \theta_{2}\right)-\left(\mathfrak{a}_{1}+\mathfrak{a}_{2} / 2 \mathfrak{a}_{1} \mathfrak{a}_{2}\right)}\right) \\
\leq & \frac{1}{2}\left[\Omega\left(\frac{1}{\left(1 / \theta_{1}\right)+\left(1 / \theta_{2}\right)-\left(\left(\eta / \mathfrak{a}_{1}\right)+\left(1-\eta / \mathfrak{a}_{2}\right)\right)}\right)+\Omega\left(\frac{1}{\left(1 / \theta_{1}\right)+\left(1 / \theta_{2}\right)-\left(\left(1-\eta / \mathfrak{a}_{1}\right)+\left(\eta / \mathfrak{a}_{2}\right)\right)}\right)\right] \\
& \cdot \frac{1}{2}\left[\omega\left(\frac{1}{\left(1 / \theta_{1}\right)+\left(1 / \theta_{2}\right)-\left(\left(\eta / \mathfrak{a}_{1}\right)+\left(1-\eta / \mathfrak{a}_{2}\right)\right)}\right)\right. \\
+ & \left.\omega\left(\frac{1}{\left(1 / \theta_{1}\right)+\left(1 / \theta_{2}\right)-\left(\left(1-\eta / \mathfrak{a}_{1}\right)+\left(\eta / \mathfrak{a}_{2}\right)\right)}\right)\right] \\
\leq & \frac{1}{4}\left[\Omega\left(\frac{1}{\left(1 / \theta_{1}\right)+\left(1 / \theta_{2}\right)-\left(\left(\eta / \mathfrak{a}_{1}\right)+\left(1-\eta / \mathfrak{a}_{2}\right)\right)}\right) \omega\left(\frac{1}{\left(1 / \theta_{1}\right)+\left(1 / \theta_{2}\right)-\left(\left(\eta / \mathfrak{a}_{1}\right)+\left(1-\eta / \mathfrak{a}_{2}\right)\right)}\right)\right] \\
& +\frac{1}{4}\left[\Omega\left(\frac{1}{\left(1 / \theta_{1}\right)+\left(1 / \theta_{2}\right)-\left(\left(1-\eta / \mathfrak{a}_{1}\right)+\left(\eta / \mathfrak{a}_{2}\right)\right)}\right)\right. \\
& \left.\cdot \omega\left(\frac{1}{\left(1 / \theta_{1}\right)+\left(1 / \theta_{2}\right)-\left(\left(1-\eta / \mathfrak{a}_{1}\right)+\left(\eta / \mathfrak{a}_{2}\right)\right)}\right)\right]+\frac{\eta(1-\eta)}{2} M\left(\theta_{1}, \theta_{2}, \mathfrak{a}_{1}, \mathfrak{a}_{2}\right)+\frac{2 \eta^{2}-2 \eta+1}{4} N\left(\theta_{1}, \theta_{2}, \mathfrak{a}_{1}, \mathfrak{a}_{2}\right) .
\end{aligned}
$$

Thus,

$$
\begin{aligned}
\Omega( & \left.\frac{1}{\left(1 / \theta_{1}\right)+\left(1 / \theta_{2}\right)-\left(\mathfrak{a}_{1}+\mathfrak{a}_{2} / 2 \mathfrak{a}_{1} \mathfrak{a}_{2}\right)}\right) \omega\left(\frac{1}{\left(1 / \theta_{1}\right)+\left(1 / \theta_{2}\right)-\left(\mathfrak{a}_{1}+\mathfrak{a}_{2} / 2 \mathfrak{a}_{1} \mathfrak{a}_{2}\right)}\right) \\
\leq & \frac{1}{4}\left[\Omega\left(\frac{1}{\left(1 / \theta_{1}\right)+\left(1 / \theta_{2}\right)-\left(\left(\eta / \mathfrak{a}_{1}\right)+\left(1-\eta / \mathfrak{a}_{2}\right)\right)}\right) \omega\left(\frac{1}{\left(1 / \theta_{1}\right)+\left(1 / \theta_{2}\right)-\left(\left(\eta / \mathfrak{a}_{1}\right)+\left(1-\eta / \mathfrak{a}_{2}\right)\right)}\right)\right] \\
& +\frac{1}{4}\left[\Omega\left(\frac{1}{\left(1 / \theta_{1}\right)+\left(1 / \theta_{2}\right)-\left(\left(1-\eta / \mathfrak{a}_{1}\right)+\left(\eta / \mathfrak{a}_{2}\right)\right)}\right)\right. \\
& \left.\cdot \omega\left(\frac{1}{\left(1 / \theta_{1}\right)+\left(1 / \theta_{2}\right)-\left(\left(1-\eta / \mathfrak{a}_{1}\right)+\left(\eta / \mathfrak{a}_{2}\right)\right)}\right)\right]+\frac{\eta(1-\eta)}{2} M\left(\theta_{1}, \theta_{2}, \mathfrak{a}_{1}, \mathfrak{a}_{2}\right)+\frac{2 \eta^{2}-2 \eta+1}{4} N\left(\theta_{1}, \theta_{2}, \mathfrak{a}_{1}, \mathfrak{a}_{2}\right) .
\end{aligned}
$$


Multiplying by $\exp (-\rho \eta)$ on both sides of (63) and then integrating with respect to $\eta \in[0,1]$, we obtain

$$
\begin{aligned}
\frac{1-}{\rho} \exp (-\rho) & \Omega\left(\frac{1}{\left(1 / \theta_{1}\right)+\left(1 / \theta_{2}\right)-\left(\mathfrak{a}_{1}+\mathfrak{a}_{2} / 2 \mathfrak{a}_{1} \mathfrak{a}_{2}\right)}\right) \omega\left(\frac{1}{\left(1 / \theta_{1}\right)+\left(1 / \theta_{2}\right)-\left(\mathfrak{a}_{1}+\mathfrak{a}_{2} / 2 \mathfrak{a}_{1} \mathfrak{a}_{2}\right)}\right) \\
\leq & \frac{1}{4} \int_{0}^{1} \exp (-\rho \eta)\left[\Omega\left(\frac{1}{\left(1 / \theta_{1}\right)+\left(1 / \theta_{2}\right)-\left(\left(\eta / \mathfrak{a}_{1}\right)+\left(1-\eta / \mathfrak{a}_{2}\right)\right)}\right) \omega\left(\frac{1}{\left(1 / \theta_{1}\right)+\left(1 / \theta_{2}\right)-\left(\left(\eta / \mathfrak{a}_{1}\right)+\left(1-\eta / \mathfrak{a}_{2}\right)\right)}\right)\right] d \eta \\
& +\frac{1}{4} \int_{0}^{1} \exp (-\rho \eta)\left[\Omega\left(\frac{1}{\left(1 / \theta_{1}\right)+\left(1 / \theta_{2}\right)-\left(\left(1-\eta / \mathfrak{a}_{1}\right)+\left(\eta / \mathfrak{a}_{2}\right)\right)}\right) \omega\left(\frac{1}{\left(1 / \theta_{1}\right)+\left(1 / \theta_{2}\right)-\left(\left(1-\eta / \mathfrak{a}_{1}\right)+\left(\eta / \mathfrak{a}_{2}\right)\right)}\right)\right] d \eta \\
& +M\left(\theta_{1}, \theta_{2}, \mathfrak{a}_{1}, \mathfrak{a}_{2}\right) \int_{0}^{1} \exp (-\rho \eta) \frac{\eta(1-\eta)}{2} d \eta+N\left(\theta_{1}, \theta_{2}, \mathfrak{a}_{1}, \mathfrak{a}_{2}\right) \int_{0}^{1} \exp (-\rho \eta) \frac{2 \eta^{2}-2 \eta+1}{4} d \eta \\
= & \frac{\alpha \mathfrak{a}_{1} \mathfrak{a}_{2}}{4\left(\mathfrak{a}_{2}-\mathfrak{a}_{1}\right)}\left[I_{\left(1 / \theta_{1}+1 / \theta_{2}-1 / \mathfrak{a}_{1}\right)}^{\alpha}(\Omega \omega \circ \mathfrak{g})\left(\frac{1}{\theta_{1}}+\frac{1}{\theta_{2}}-\frac{1}{\mathfrak{a}_{2}}\right)+I_{\left(1 / \theta_{1}+1 / \theta_{2}-1 / \mathfrak{a}_{2}\right)}^{\alpha}(\Omega \omega \circ \mathfrak{g})\left(\frac{1}{\theta_{1}}+\frac{1}{\theta_{2}}-\frac{1}{\mathfrak{a}_{1}}\right)\right] \\
& +\frac{\rho-2+\exp (-\rho)(\rho+2)}{2 \rho^{3}} M\left(\theta_{1}, \theta_{2}, \mathfrak{a}_{1}, \mathfrak{a}_{2}\right)+\frac{\rho^{2}-2 \rho+4-\left(\rho^{2}+2 \rho+4\right) \exp (-\rho)}{4 \rho^{3}} N\left(\theta_{1}, \theta_{2}, \mathfrak{a}_{1}, \mathfrak{a}_{2}\right)
\end{aligned}
$$

Thus,

$$
\begin{aligned}
& \Omega\left(\frac{1}{\left(1 / \theta_{1}\right)+\left(1 / \theta_{2}\right)-\left(\mathfrak{a}_{1}+\mathfrak{a}_{2} / 2 \mathfrak{a}_{1} \mathfrak{a}_{2}\right)}\right) \omega\left(\frac{1}{\left(1 / \theta_{1}\right)+\left(1 / \theta_{2}\right)-\left(\mathfrak{a}_{1}+\mathfrak{a}_{2} / 2 \mathfrak{a}_{1} \mathfrak{a}_{2}\right)}\right) \\
& \leq \frac{1-\alpha}{4[1-\exp (-\rho)]}\left[I_{\left(1 / \theta_{1}+1 / \theta_{1}-1 / \mathfrak{a}_{1}\right)}^{\alpha}(\Omega \omega \circ \mathfrak{g})\left(\frac{1}{\theta_{1}}+\frac{1}{\theta_{2}}-\frac{1}{\mathfrak{a}_{2}}\right)+I_{\left(1 / \theta_{1}+1 / \theta_{1}-1 / \mathfrak{a}_{2}\right)}^{\alpha}(\Omega \omega \circ \mathfrak{g})\left(\frac{1}{\theta_{1}}+\frac{1}{\theta_{2}}-\frac{1}{\mathfrak{a}_{1}}\right)\right] \\
& \quad+\frac{\rho-2+\exp (-\rho)(\rho+2)}{2 \rho^{2}[1-\exp (-\rho)]} M\left(\theta_{1}, \theta_{2}, \mathfrak{a}_{1}, \mathfrak{a}_{2}\right)+\frac{\rho^{2}-2 \rho+4-\left(\rho^{2}+2 \rho+4\right) \exp (-\rho)}{4 \rho^{2}[1-\exp (-\rho)]} N\left(\theta_{1}, \theta_{2}, \mathfrak{a}_{1}, \mathfrak{a}_{2}\right)
\end{aligned}
$$

Remark 10. If we take $\mathfrak{a}_{1}=\theta_{1}$ and $\mathfrak{a}_{2}=\theta_{2}$ in Theorem 12, Remark 11. For $\alpha \longrightarrow 1$, we have then we get Theorem 12 in [24].

$$
\begin{aligned}
\lim _{\alpha \longrightarrow 1} \frac{1-\alpha}{2[1-\exp (-\rho)]} & =\frac{\mathfrak{a}_{1} \mathfrak{a}_{2}}{2\left(\mathfrak{a}_{2}-\mathfrak{a}_{1}\right)}, \\
\lim _{\alpha \longrightarrow 1} \frac{\rho-2+\exp (-\rho)(\rho+2)}{\rho^{3}} & =\frac{1}{6} \\
\lim _{\alpha \longrightarrow 1} \frac{\rho^{2}-2 \rho+4-\left(\rho^{2}+2 \rho+4\right) \exp (-\rho)}{2 \rho^{2}[1-\exp (-\rho)]} & =\frac{1}{3} .
\end{aligned}
$$


Under the assumptions of Theorem 12 with $\alpha=1$, we have

$$
\begin{aligned}
\frac{\mathfrak{a}_{1} \mathfrak{a}_{2}}{\mathfrak{a}_{2}-\mathfrak{a}_{1}} \int_{1 / \theta_{1}+1 / \theta_{2}-1 / \mathfrak{a}_{1}}^{1 / \theta_{1}+1 / \theta_{2}-1 / \mathfrak{a}_{2}} \frac{\Omega(\varkappa)}{\varkappa^{2}} \omega(\varkappa) \mathrm{d} \varkappa & \leq \frac{M\left(\theta_{1}, \theta_{2}, \mathfrak{a}_{1}, \mathfrak{a}_{2}\right)}{3}+\frac{N\left(\theta_{1}, \theta_{2}, \mathfrak{a}_{1}, \mathfrak{a}_{2}\right)}{6}, \\
& 2 \Omega\left(\frac{1}{\left(1 / \theta_{1}\right)+\left(1 / \theta_{2}\right)-\left(\mathfrak{a}_{1}+\mathfrak{a}_{2} / 2 \mathfrak{a}_{1} \mathfrak{a}_{2}\right)}\right) \omega\left(\frac{1}{\left(1 / \theta_{1}\right)+\left(1 / \theta_{2}\right)-\left(\mathfrak{a}_{1}+\mathfrak{a}_{2} / 2 \mathfrak{a}_{1} \mathfrak{a}_{2}\right)}\right) \\
& \leq \frac{\mathfrak{a}_{1} \mathfrak{a}_{2}}{\mathfrak{a}_{2}-\mathfrak{a}_{1}} \int_{1 / \theta_{1}+1 / \theta_{2}-1 / \mathfrak{a}_{1}}^{1 / \theta_{1}+1 / \theta_{2}-1 / \mathfrak{a}_{2}} \frac{\Omega(\varkappa)}{\varkappa^{2}} \omega(\varkappa) \mathrm{d} \varkappa+\frac{M\left(\theta_{1}, \theta_{2}, \mathfrak{a}_{1}, \mathfrak{a}_{2}\right)}{6}+\frac{N\left(\theta_{1}, \theta_{2}, \mathfrak{a}_{1}, \mathfrak{a}_{2}\right)}{3} .
\end{aligned}
$$

Remark 12. If $\alpha=1, \mathfrak{a}_{1}=\theta_{1}$, and $\mathfrak{a}_{2}=\theta_{2}$ in Theorem 12, then we obtain inequalities (9) and (10) for harmonically convex functions proved by Chen and $\mathrm{Wu}$ in [13].

\section{Concluding Remarks and Future Directions}

In this study, we introduce for the first time the unified variants of Hermite-Hadamard, Fejér-Hadamard, and Pachpatte-Mercer-type inequalities for harmonically convex functions for fractional integral operators with the exponential kernel. New integral identity involving fractional integral operators with exponential kernel is developed. A compact analysis of newly obtained results and their connections is explained in Remarks 2-12. As special cases, we get Hermite-Hadamard, Fejér-Hadamard, and Pachpatte-Mercer-type inequalities for classical calculus with explicit boundary values. Some particular cases reflect the related existing results. One of the direct impact and utilization of the results extracted in this paper is to obtain inequalities involving following new fractional integral operators containing Mittag-Leffler nonsingular kernels:

$$
\begin{aligned}
& \rrbracket_{\mathfrak{a}_{1}}^{\alpha} \Omega(\varkappa)=\frac{1}{\alpha} \int_{\mathfrak{a}_{1}}^{\varkappa} E_{\alpha, 1}\left(-\frac{1-\alpha}{\alpha}(\varkappa-u)^{\alpha}\right) \Omega(u) \mathrm{d} u, \quad \varkappa>\mathfrak{a}_{1}, \\
& \square_{\mathfrak{a}_{2}}^{\alpha} \Omega(\varkappa)=\frac{1}{\alpha} \int_{\varkappa}^{\mathfrak{a}_{2}} E_{\alpha, 1}\left(-\frac{1-\alpha}{\alpha}(u-\varkappa)^{\alpha}\right) \Omega(u) \mathrm{d} u, \quad \varkappa<\mathfrak{a}_{2},
\end{aligned}
$$

for $\alpha \in(0,1)$ and $\Omega \in L\left(\mathfrak{a}_{1}, \mathfrak{a}_{2}\right)$, where $E_{\alpha, v}(\xi)$ is a MittagLeffler-type function:

$$
E_{\alpha, \nu}(\xi)=\sum_{\iota=0}^{\infty} \frac{\xi^{l}}{\Gamma(\alpha \iota+\nu)} .
$$

These integral inequalities may be helpful in the circumstances where upper and lower bounds matter for fractional integral operators involving nonlocal kernels. It is natural to investigate such results for other general convexities like harmonically $h$-convex functions introduced by Noor et al. in [30]. Also, it is interesting to construct such inequalities over fractal domains where we may get optimal and sharp local fractional integral inequalities involving Mittag-Leffler kernel.

\section{Data Availability}

No data were used to support this study.

\section{Conflicts of Interest}

The authors declare that they have no conflicts of interest.

\section{Acknowledgments}

Abdullah M. Alsharif was supported by Taif University Researchers Supporting Project (TURSP-2020/96), Taif University, Taif, Saudi Arabia.

\section{References}

[1] D. S. Mitrinović, J. E. Pečarić, and A. M. Fink, "Classical and new inequalities in analysis," Mathematics and its Applications (East European Series), Kluwer Academic Publishers Group, Dordrecht, Netherlands, 1993.

[2] S. S. Dragomir and C. E. M. Pearce, Selected Topics on Hermite-Hadamard Inequalities and Applications, RGMIA Monographs, Victoria University, Melbourne, Australia, 2000.

[3] C. P. Niculescu and L. E. Persson, "Convex functions and their applications: a contemporary approach," CMS Books in Mathematics/Ouvrages de Math ematiques de la SMC, Springer, New York, NY, USA, 2nd edition, 2018.

[4] M. Khan, Z. Al-sahwi, and Y.-M. Chu, "New estimations for shannon and zipf-mandelbrot entropies," Entropy, vol. 20, no. 8, p. 608, 2018.

[5] S. Khan, M. A. Khan, S. I. Butt, and Y. M. Chu, "A new bound for the Jensen gap pertaining twice differentiable functions with applications," Advances in Difference Equations, vol. 2020, p. 333, 2020.

[6] S. I. Butt, L. Horváth, D. Pečarić, and J. Pečarić, "Cyclic improvements of Jensen's inequalities (cyclic inequalities in information theory)," Monographs in Inequalities, Element, Zagreb, Croatia, 2020.

[7] A. M. Mercer, "A variant of jensen's inequality," Journal of Inequalities in Pure and Applied Mathematics, vol. 4, no. 4, p. 73, 2003.

[8] M. Kian and M. Moslehian, "Refinements of the operator Jensen-Mercer inequality," The Electronic Journal of Linear Algebra, vol. 26, pp. 742-753, 2013.

[9] E. Anjidani, "Jensen-Mercer operator inequalities involving superquadratic functions," Mediterranean Journal of Mathematics, vol. 15, p. 31, 2018. 
[10] H. R. Moradi and S. Furuichi, "Improvement and generalization of some Jensen-Mercer-type inequalities," Journal of Mathematical Inequalities, vol. 14, no. 2, pp. 377-383, 2020.

[11] Í. Íşcan, "Hermite-Hadamard type inequalities for harmonically convex functions," Hacettepe Journal of Mathematics and Statistics, vol. 43, no. 6, pp. 935-942, 2014.

[12] F. Chen and S. Wu, "Fejer Hermite-Hadamard type inqequalities for harmonically convex functions," Journal of Applied Mathametics, vol. 2014, Article ID 386806, 2014.

[13] F. Chen and S. Wu, "Some Hermite-Hadamard type inequalities for harmonically s-convex functions," The Scientific World Journal, vol. 2014, Article ID 279158, 2014.

[14] R. Gorenflo, "Fractional calculus," in Fractals and Fractional Calculus in Continuum Mechanics, A. Carpinteri and F. Mainardi, Eds., Springer-Verlag, New York, NY, USA, 1997.

[15] A. A. Kilbas, H. M. Srivastava, and J. J. Trujillo, Theory and Applications of Fractional Differential Equations, Elservier, Amsterdam, Netherlands, 2006.

[16] S. Rashid, Z. Hammouch, D. Baleanu, and Y.-M. Chu, "New generalizations in the sense of the weighted non-singular fractional integral operator," Fractals, vol. 28, no. 8, Article ID 2040003, 2020.

[17] S. I. Butt, S. Yousaf, A. O. Akdemir, and M. A. Dokuyucu, "New Hadamard-type integral inequalities via a general form of fractional integral operators," Chaos, Solitons \& Fractals, vol. 148, Article ID 111025, 2021.

[18] X. Wu, J. R. Wang, and J. Zhang, "Hermite-Hadamard-type inequalities for convex functions via the fractional integrals with exponential kernel," Mathematics, vol. 7, no. 845, pp. 1-12, 2019.

[19] A. O. Akdemir, S. I. Butt, M. Nadeem, and M. A. Ragusa, "New general variants of Chebyshev type inequalities via generalized fractional integral operators," Mathematics, vol. 9, no. 2, p. 122, 2021.

[20] Í. Íşcan and S. Wu, "Hermite-Hadamard type inequalities for harmonically convex function via fractional integrals," Applied Mathametics Computation, vol. 238, pp. 237-244, 2014.

[21] Í. Íşcan and M. Kunt, "Hermite-Hadamard-Fejér type inequalities for harmonically convex functions via fractional integrals," RGMIA Research Report Collection, vol. 18, pp. 1-16, 2015.

[22] K. Mehrez and P. Agarwal, "New Hermite-Hadamard type integral inequalities for convex functions and their applications," Journal of Computer Applied Mathametics, vol. 350, pp. 274-285, 2019.

[23] B. Ahmad, A. Alsaed, M. Kirane, and B. T. Torebek, "HermiteHadamard, Hermite-Hadamard-Fejér, Dragomir-Agarwal and Pachpatte type inequalities for convex functions via new fractional integrals," Journal of Computer Applied Mathametics, vol. 353, pp. 120-129, 2019.

[24] S. Rashid, D. Baleanu, and Y.-M. Chu, "Some new extensions for fractional integral operator having exponential in the kernel and their applications in physical systems," Open Physics, vol. 18, no. 1, pp. 478-491, 2020.

[25] H.-H. Chu, S. Rashid, Z. Hammouch, and Y. M. Chu, "New fractional estimates for Hermite-Hadamard-Mercer's type inequalities," Alexandria Engineering Journal, vol. 59, no. 5, pp. 3079-3089, 2020.

[26] S. I. Butt, M. Umar, S. Rashid, A. O. Akdemir, and Y. M. Chu, "New Hermite-Mercer type inequalities via $\mathrm{k}$-fractional integrals," Advances in Differential Equations, vol. 2020, Article ID 635, 2020.
[27] S. I. Butt, J. Nasir, S. Qaisar, and K. M. Abualnaja, "k-Fractional variants of Hermite-Mercer-type inequalities via s-convexity with applications," Journal of Function Spaces, vol. 2021, Article ID 5566360, 2021.

[28] I. A. Baloch, A. A. Mughal, Y.-M. U. Haq, and M. D. L. Sen, “A variant of Jensen-type inequality and related results for harmonic convex functions," AIMS Mathematics, vol. 5, no. 6, pp. 6404-6418, 2020.

[29] I. A. Baloch, A. A. Mughal, Y. M. Chu, A. U. Haq, and M. D. L. Sen, "Improvement and generalization of some results related to the class of harmonically convex functions and applications," Journal of Mathametics Computer Science, vol. 22, no. 3, pp. 282-294, 2021.

[30] M. A. Noor, K. I. Noor, M. U. Awan, and S. Costache, "Improvement and generalization of some results related to the class of harmonically h-convex functions and applications," U.P.B. Scientific Bulletin Series A, vol. 77, no. 1, pp. 1-16, 2015. 